\title{
Dynamical states and the conventionality of (non-) classicality
}

\author{
Alexander Wilce \\ Department of Mathematics \\ Susquehanna University
}

December 13, 2019

\begin{abstract}
Itamar Pitowsky long championed the view that quantum mechanics (QM) is best understood as a non-classical probability theory. Here, I want to offer some modest caveats. One is that QM is best seen, not as a new probability theory, but as something narrower, namely, a particular probabilistic theory - roughly, a class of probabilistic models, selected from within a much more general framework. It is this general framework that, if anything, deserves to be regarded as a "non-classical" probability theory. However, as I will also argue, this framework represents a very conservative extension of classical probability theory, essentially just eliding a tacit, and contingent, assumption in the latter that measurements or experiments can always be performed together. Moreover, for individual probabilistic models, and even for probabilistic theories, the distinction between "classical" and a "non-classical" is largely a conventional one, bound up with the question of what one means by the state of a system. In fact, for systems with a high degree of symmetry, including quantum mechanics, it is possible to interpret general probabilistic models as having a perfectly classical probabilistic structure, but an additional dynamical structure: states, rather than corresponding simply to probability measures, are represented as certain probability measure-valued functions on the system's symmetry group, and thus, as fundamentally dynamical objects. Conversely, a classical probability space equipped with reasonably well-behaved family of such "dynamical states" can be interpreted as a generalized probabilistic model in a canonical way. It is noteworthy that this "dynamical" representation is not a conventional hiddenvariables representation, and the question of what one means by "non-locality" in this setting is not entirely straightforward.
\end{abstract}

\section{Introduction}

Itamar Pitowsky long championed the idea (also associated with Jeff Bub, among others) that quantum theory is best viewed as a non-classical probability theory [21]. This position is strongly motivated by the fact that the mathematical apparatus of quantum mechanics can largely be reduced to the statement that the binary observables — the physically measurable, $\{0,1\}$-valued quantitites — associated with a quantum system are represented by the projection operators on a Hilbert space, or, more generally, the projections in a von Neumann algebra, in such a way that commuting families of projections can be jointly measured and mutually orthogonal projections are mutually exclusive. ${ }^{1}$ In other words, mathematically, quantum theory is what one gets when one replaces the Boolean algebras of classical probability theory with projection lattices. It is very natural to conclude from this that quantum theory simply is a "non-classical" or "non-commutative" probability theory.

\footnotetext{
${ }^{1}$ Given this, Gleason's Theorem, as extended by Christensen and Yeadon, identifies the system's states with states (positive, normalized linear functionals) on that same von Neumann algebra, and Wigner's Theorem then identifies the possible dynamics with one-parameter groups of unitary elements of that algebra. For the details, see [24].
} 
This point of view has many attractions, not least that it more or less dissolves what Pitowsky called the "big" measurement problem [7, 21]. It also allows one to ask why quantum mechanics has the particular non-classical probabilistic structure that it does. The past decade or two has seen a good deal of progress on this question, at least as it pertains to finite-dimensional quantum systems [5, 9, 13, 19, 28], The upshot is that the probabilistic apparatus of finite-dimensional QM can be reconstructed from various different packages of assumptions, all having a broadly probabilistic character, lending some further support to Pitowsky's position.

Here, I want to offer some modest caveats. The first is that how we understand Pitowski's thesis depends on how we understand probabability theory itself. On the view that I favor, quantum mechanics (henceforth, QM) is best seen, not as replacement for general classical probability theory, but rather, as a specific class of probabilistic models - a probabilistic theory - defined within a much broader framework, in which individual models are defined by essentially arbitrary sets of basic measurements and states, with the latter assigning probabilities (in an elementary sense) to outcomes of the former. It is this framework that, if anything, counts as a "non-classical" probability theory — but in fact, I think we should regard, and refer to, this general framework, simply as probability theory, without any adjective. Since it outruns what's usually studied under that heading, I settle here for the term general probability theory. ${ }^{2}$ Within this framework, in addition to QM, we can identify classical probability theory, in the strict Kolmogorovian sense, as a special, and, I will argue, equally contingent, probabilistic theory.

However - and this is my second point - even as applied to individual probabilistic models, or, indeed, individual probabilistic theories, the distinction between "classical" and "non-classical" is not so clear cut. One standard answer to the question of what makes the probabilistic apparatus of QM "non-classical" is that its state space (the set of density operators on a Hilbert space, or the set of states on a non-abelian von Neumann algebra, depending on how general we want to be) is not a simplex: the same state can be realized as a mixture - a convex combination - of pure states in many different ways. Another, related, answer is that, whereas in classical probability theory any two measurements (or, if you prefer, experiments) can effectively be performed jointly, in QM this is not the case.

Neither of these answers is really satisfactory. In the first place, it is perfectly within the scope of standard classical probability theory to consider restricted sets of probability measures and random variables on a given measurable space: the former need not be simplices, and the latter need not be closed under the formation of joint random variables. We might also wish to allow "unsharp" random variables having some intrinsic randomness (represented, depending on one's taste, by Markov kernels or by response-function valued measures). We might try to identify as "broadly classical" just those probabilistic models arising in this way, from a measurable space plus some selection of probability measures and (possibly unsharp) random variables. But once we open the door this far, it's difficult to keep it on its hinges. This is because there exist straightforward, well known, and in a sense even canonical, recipes for representing essentially any "non-classical" probabilistic model in terms of such a broadly classical one, either by treating the state space as a suitable quotient of a simplex (in the manner of the Beltrametti-Bugajski representation of quantum mechanics), or by introducing contextual "hidden variables". In fact, for systems with a high degree of symmetry, including finite-dimensional quantum systems, there is another, less well known, but equally straightforward classical representation: the probabilistic structure is simply that of a single reference observable, and hence, entirely classical; states, however, rather than corresponding simply to probability measures, are represented by probability measure-valued functions on the system's symmetry group, and thus, as fundamentally dynamical objects. Conversely, a classical probability space equipped with a family of suitably covariant such "dynamical states" — what I call a dynamical classical model can be interpreted as a generalized probabilistic model in a canonical way.

At the level of individual probabilistic models, then, it seems that the distinction between classical and nonclassical is, at least mathematically, more or less one of convention (and, pragmatically, of convenience). There is, of course, another consideration in play: that of locality. For probabilistic theories involving a

\footnotetext{
${ }^{2}$ The framework in question is broadly equivalent to that of "generalized probabilistic theories". Luckily, both phrases can go by the acronym GPT.
} 
notion of composite systems that admits entangled states - including, of course, quantum theory - the classical representations alluded to above are all manifestly non-local in one sense or another. One reading of Bell's Theorem is that, for such probabilistic theories, there is a necessary tension between "classicality" and locality. Indeed, one standard response to the existence of various classical representations is to dismiss them precisely because they are non-local. But locality is very much a physical constraint, rather than a "law of thought". Thus, we should be careful not to regard the probabilistic apparatus of quantum mechanics as a successor to classical probability theory as a general account of reasoning in the face of uncertainty. Rather, it is a particular, contingent, probabilistic physical theory, expressible in terms of a much broader generalized probability theory - a framework that we may or may not prefer to regard as non-classical, but which is in any event at most a very conservative generalization of classical probability theory. It is a collateral aim of this Chapter to argue for this expansive view of what probability theory $i s$.

A brief outline of this Chapter is as follows. In section 2, I give a condensed introduction to the framework - or rather, one particular version of the framework - of general(ized) probability theory, along the lines of [4]. It is another collateral aim of this paper to advertise this framework as offering some additional measure of clarity to discussions of foundational issues in quantum theory. In section 3, I ask how one ought to characterize classical probabilistic models in this framework, and review several more or less canonical ways in which an arbitrary probabilistic model can be interpreted in terms of a classical one. There is little here that is new, except possibly some streamlining of the material. In section 4 , I introduce the dynamical classical models alluded to above. In section 5, I very briefly discuss some issues of locality, entanglement and so forth in this context, and in Section 6, I gather a few concluding thoughts. I have placed some technical material in a series of appendices. Appendix A establishes a folk-result, to the effect that a probabilistic model in which any two measurements are compatible in the sense of admitting a common refinement, is essentially classical. Appendix B gathers some straightforward observations about what are called semiclassical test spaces. Appendix C concerns a construction that can be used to generate large numbers of highly symmetric probabilistic models, or, equivalently, dynamical classical models. This follows, but in some respects generalizes, material in [27]. To keep measure-theoretic details to a minimum, I focus mainly (though not exclusively) on probabilistic models in which there is a finite upper bound on the number of distinct outcomes in a basic measurement.

Terminology: In the balance of this chapter, I will use the adjective classical sometimes in a broad and informal way, sometimes in a more technical sense, and sometimes ironically. I hope that context, occasionally aided by scare-quotes, will help make my meaning clear in each case. The term classical representation is also to be understood broadly and somewhat informally, to mean any mathematical representation of a one probabilistic model or theory in terms of another such model or theory, the latter being in some reasonable sense classical. At two points, my mathematical usage may be slightly nonstandard: first, by a measurable space, I will always mean a pair $(S, \Sigma)$ where $S$ is a set and $\Sigma$ is an algebra, but not necessarily a $\sigma$-algebra, of subsets of $S$; and by a measure on $S$, unless otherwise specified I mean a finitely-additive measure. Secondly, by a discrete Markov kernel I mean a function $p: S \times T \rightarrow[0,1]$, where $S$ and $T$ are sets, possibly infinite, such that $\sum_{y \in T} p(x, y)=1$ for every $x \in S$, where the sum is meant in the unordered sense. Otherwise, my usage will be fairly standard. Terms likely to be unfamiliar to most readers are defined when introduced.

\section{Probability theory vs probabilistic theories}

This section provides a brief but self-contained tutorial on the mathematical framework that I favor for a general probability theory. This is a slight elaboration of a formalism developed by D. J. Foulis and C. H. Randall; see, e.g., [11, 12]. A more complete survey can be found in [4]. 


\subsection{Test spaces, probability weights, and probabilistic models}

In many introductory treatments of probability theory, a probabilistic model is defined as a pair $(E, p)$ where $E$ is the outcome-set of some experiment, and $p$ is a probability weight on $E$, meant to reflect the actual statistical behaviour of the experiment. An obvious generalization is to allow $p$ to vary, i.e., to consider pairs $(E, \Omega)$ where $\Omega$ is some set of possible probability weights. For example, if $E=\{0,1\}^{n}$, we might want to restrict attention to binomial probability weights with various probabilities of success.

An only slightly less obvious generalization is to allow $E$, too, to vary:

Definition 2.1 (Test spaces) A test space is a set $\mathcal{M}$ of non-empty sets $E, F, \ldots$. , understood as the outcome-sets of various experiments or, as we'll say, tests. If $X:=\bigcup \mathcal{M}$ is the set of all outcomes of all tests in $\mathcal{M}$, a probability weight on $\mathcal{M}$ is a function $\alpha: X \rightarrow[0,1]$ such that $\sum_{x \in E} \alpha(x)=1$ for every $E \in \mathcal{M}$.

I will write $\operatorname{Pr}(\mathcal{M})$ for the set of all probability weights on a test space $\mathcal{M}$. Note that $\operatorname{Pr}(\mathcal{M})$ is a convex subset of $\mathbb{R}^{X}$ : any weighted average $p \alpha+(1-p) \beta$ of probability weights $\alpha, \beta \in \operatorname{Pr}(\mathcal{M})$ is again a probability weight on $\mathcal{M}$.

Definition 2.2 (Probabilistic models) A probabilistic model is a pair $A=(\mathcal{M}, \Omega)$ where $\mathcal{M}$ is a test space and $\Omega \subseteq \operatorname{Pr}(\mathcal{M})$ is a convex set of probability weights on $\mathcal{M}$, which we call the states of the model. Extreme points of $\Omega$ are called pure states.

It will be convenient to denote a probabilistic model by a single letter $A, B$, etc. When doing so, I write, e.g., $A=(\mathcal{M}(A), \Omega(A))$. The assumption that $\Omega(A)$ is convex is meant to reflect the possibility of randomizing the preparation of states; that is, if we have some procedure that produces state $\alpha$ and another that produces state $\beta$, we could flip a suitably biased coin to product the state $p \alpha+(1-p) \beta$ for any $0 \leq p \leq 1$. It is also common to equip a probabilistic model $A$ with a preferred group $G(A)$ of "physical transformations" $[10,13,19]$, and I will also do so in Section 4 . Until then, however, this extra structure will not be necessary. Finally, I will suppose in what follows that $\Omega$ contains sufficiently many probability weights to separate points of $X$ and that $\mathcal{M}$ contains sufficiently many tests (and hence $X$, sufficiently many outcomes) to distinguish the states in $\Omega$ (since otherwise, one would presumably just identify outcomes, respectively states, that are not distinguishable). Another condition that I will often (but not always) impose is that, for every outcome $x \in X(A)$, there exists at least one probability weight $\alpha \in \Omega(A)$ with $\alpha(x)=1$. A model satisfying this condition is said to be unital.

Notice that we permit tests $E, F \in \mathcal{M}$ to intersect, that is, to share outcomes. From a certain point of view, this is absurd: when we perform a measurement, we usually retain a record of what measurement we've made! Nevertheless, we may have good reasons to want to identify outcomes from distinct tests, and to demand that physically meaningful operations on outcomes - symmetries, probability assignments, etc. - respect these identifications. The usual term for this is non-contextuality. In most of the probabilistic models one encounters in practice, such outcome-identifications are in fact made, and in any event, it is mathematically more general to allow tests to overlap than to forbid them to do so. Moreover, contextual probability weights can easily be accommodated by way of the following construction.

Definition 2.3 (Semi-classical test spaces) A test space $\mathcal{M}$ is semi-classical iff $E \cap F=\emptyset$ for all distinct tests $E, F \in \mathcal{M}$. The semi-classical cover of an arbitrary test space $\mathcal{M}$ is the test space

$$
\widetilde{\mathcal{M}}=\{\widetilde{E} \mid E \in \mathcal{M}\},
$$

where, for $E \in \mathcal{M}, \widetilde{E}=\{(x, E) \mid x \in E\}$. 
The outcome-set of $\widetilde{\mathcal{M}}$ is thus $\widetilde{X}=\{(x, E) \mid x \in E \in \mathcal{M}\}$, i.e., the the coproduct of the sets $E \in \mathcal{M}$. "Contextual" probability weights on $\mathcal{M}$ are best regarded as probability weights on $\widetilde{\mathcal{M}}$. In general $\operatorname{Pr}(\widetilde{\mathcal{M}})$ will be very much larger than $\operatorname{Pr}(\mathcal{M})$; indeed, if $\mathcal{M}$ contains infinitely many tests, $\operatorname{Pr}(\widetilde{\mathcal{M}})$ will be infinite dimensional, even if $\operatorname{Pr}(\mathcal{M})$ is finite-dimensional.

Definition 2.4 A probability weight $\alpha$ on $\mathcal{M}$ is dispersion-free or deterministic iff $\alpha(x) \in\{0,1\}$ for every $x \in X=\bigcup \mathcal{M}$.

One of the most obvious distinctions between the probabilistic models associated with "classical" systems and those associated with quantum-mechanical systems is that the former have an abundance of dispersionfree states, while the latter, absent superselection rules, have none at all. Notice, however, that $\widetilde{\mathcal{M}}$ always has an abundance of dispersion-free probability weights (just choose an outcome from every test).

Definition 2.5 (Locally finite test spaces) A test space $\mathcal{M}$ is locally finite iff every test $E \in \mathcal{M}$ is a finite set. Every test space is associated with a locally finite test space $\mathcal{M}^{\#}$, consisting of finite partitions of tests in $\mathcal{M}$. That is, writing $\mathcal{D}(E)$ for the set of finite partitions of a set $E$,

$$
\mathcal{M}^{\#}=\bigcup_{E \in \mathcal{M}} \mathcal{D}(E)
$$

If $\mathcal{M}$ is a test space, an event for $\mathcal{M}$ is a subset of a test (that is, an event in the standard sense pertaining to that test). We write $\mathcal{E} \boldsymbol{v}(\mathcal{M})$ for the set of all events of $\mathcal{M}$, so that $\mathcal{E} \boldsymbol{v}(\mathcal{M})=\bigcup_{E \in \mathcal{M}} \mathcal{P}(E)$. Thus, the outcome-space for $\mathcal{M}^{\#}$ is precisely the set $\mathcal{E} \boldsymbol{v}(\mathcal{M}) \backslash\{\emptyset\}$ of non-empty events.

If $\alpha$ is a probability weight on $\mathcal{M}$, then we define the probability of an event $a \in \mathcal{E} \boldsymbol{v}(\boldsymbol{M})$ by $\alpha(a)=$ $\sum_{x \in a} \alpha(x)$. This evidently defines a probability weight on $\mathcal{M}^{\#}$. However, unless $\mathcal{M}$ is locally finite to begin with, $\mathcal{M}^{\#}$ will generally support many additional "singular" probability weights. (Indeed, if $\mathcal{M}$ is semiclassical with infinite tests, one can simply choose a non-prinicipal ultrafilter on each $E \in \mathcal{M}$.)

Lemma 2.6 Let $\mathcal{M}$ be locally finite with outcome-set $X$. Then the convex set $\operatorname{Pr}(\mathcal{M})$ of probability weights on $\mathcal{M}$ is compact as a subset of $[0,1]^{X}$ (with the product topology).

Proof: Since each $E \in \mathcal{M}(A)$ is finte, the pointwise limit of a net of probability weights will continue to sum to unity over $E$. Thus, $\operatorname{Pr}(\mathcal{M})$ is closed, and hence, compact, in $[0,1]^{X}$.

It follows that if $A$ is a probabilistic model with $\mathcal{M}(A)$ locally finite, the closure of $\Omega(A)$ in $\operatorname{Pr}(\mathcal{M}(A))$ will also be compact. Because it is both mathematically convenient and operationally reasonable to do so, I shall assume from now on that, unless otherwise indicated, all probabilistic models $A$ under discussion have a locally finite test space $\mathcal{M}(A)$ and a closed, hence compact, state space $\Omega(A)$. While I will make no direct use of it, it's worth noting that the Krein-Milman Theorem now tells us that every state in $\Omega(A)$ is a limit of convex combinations of pure states.

It is often useful to consider sub-normalized positive weights on a test space $\mathcal{M}$, that is, functions $\alpha: X:=$ $\cup \mathcal{M} \rightarrow \mathbb{R}_{+}$such that $\sum_{x \in E} \alpha(x)=: r \leq 1$, independently of $E$. In fact, these are simply probability weights on a slightly larger test space:

Definition 2.7 (Adjoining a null-outcome) If $\mathcal{M}$ is a test space with outcome-space $X$, let $*$ be symbol not belonging to $X$, and define $\mathcal{M}_{+}=\{E \cup\{*\} \mid E \in \mathcal{M}\}$. 
We can think of $*$ as a "null outcome" representing the failure of a test, in a setting in which we regard this failure as meaning the same thing regardless of which test is performed. As an extreme example, $*$ might represent the destruction of the system under study. It should be clear that a probability weight on $\mathcal{M}_{+}$corresponds exactly to a sub-normalized weight on $\mathcal{M}$.

\subsection{Some Examples}

Example 2.8 (Kolmogorovian models) We can treat standard measure-theoretic probability theory within the present framework in the following way. Let $(S, \Sigma)$ be a measurable space and let $\mathcal{D}(S, \Sigma)$ be the set of finite partitions of $S$ by elements of $\Sigma$, regarded as a test space. Note that $X=\bigcup \mathcal{D}(S, \Sigma)$ is simply $\Sigma \backslash\{\emptyset\}$. A probability weight on $\mathcal{D}(S, \Sigma)$, then, is a function $p: \Sigma \backslash\{\emptyset\} \rightarrow[0,1]$ summing to unity on every finite partition of $S$ by members of $\Sigma$. It is straightforward to show that such a function, extended to all of $\Sigma$ by setting $p(\emptyset)=0$, is a finitely additive probability measure on $\Sigma$, and conversely. We can now construct a probabilistic model $(\mathcal{D}(S, \Sigma), \Omega)$ in various ways, for instance, by taking $\Omega$ to consist of all such measures, or of all $\sigma$-additive probability measures, or of probability measures absolutely continuous with respect to some given measure, or of a single probability measure of intererest, etc. I will refer to all models of the form $(\mathcal{D}(S, \Sigma), \Omega)$, where $\Omega$ is any convex set of probability measures on $(S, \Sigma)$, as Kolmogorovian. ${ }^{3}$

Note that every point $s \in S$ defines a dispersion-free probability weight on $\mathcal{D}(S, \Sigma)$, namely, the point-mass $\delta_{s}(b)=1$ if $b \in \Sigma$ with $s \in b$, and $\delta_{s}(b)=0$ otherwise. More generally, a dispersion-free probability weight on $\mathcal{D}(S, \Sigma)$ is an ultrafilter on $\Sigma$.

Example 2.9 (Quantum models) If $\mathcal{H}$ is a Hilbert space, let $X(\mathcal{H})$ denote $\mathcal{H}$ 's unit sphere and $\mathcal{M}(\mathcal{H})$, the set of maximal pairwise orthogonal subsets of $X(\mathcal{H})$, that is, the set of unordered orthonormal bases of $\mathcal{H}$. We shall call this the quantum test space associated with $\mathcal{H}$. Any density operator $W$ on $\mathcal{H}$ gives rise to a probability weight $\alpha_{W}$ on $\mathcal{M}(\mathcal{H})$, given by $\alpha_{W}(x)=\langle W x, x\rangle^{4}$. Let $\Omega(\mathcal{H})$ be the set of all probability weights on $\mathcal{M}(\mathcal{H})$ arising from density operators in this way: then $A(\mathcal{H}):=(\mathcal{M}(\mathcal{H}), \Omega(\mathcal{H}))$ is a probabilistic model representing the same quantum-mechanical system one associates with $\mathcal{H}$, but in a manner that puts its probabilistic structure in the foreground. Note that $\mathcal{H}$ is finite-dimensional, $\Omega(\mathcal{H})$ is compact, and vice versa.

While the model above is mathematically attractive, it is more usual to identify measurement outcomes with projection operators. The projective quantum test space consists of sets of projections $p_{i}$ on $\mathcal{H}$ summing to $\mathbf{1}$. If $\rho$ is a density operator on $\mathcal{H}$, we obtain a probability weight (also denoted by $\rho$ ) given by $\rho(p)=\operatorname{Tr}(\rho p)$. Letting $\Omega$ consist of all such probability weights, we obtain what we can call the projective quantum model. More generally still, if $\mathfrak{A}$ is a von Neumann algebra (or, for that matter, any ring with identity), the collection $\mathcal{M}(\mathfrak{A})$ of sets $E$ of projections $p \in \mathfrak{A}$ with $\sum_{p \in E} p=\mathbf{1}$ is a test space, on which every state on $\mathfrak{A}$ gives rise to a probability weight by evaluation. In this case, we might identify $\Omega$ with the state space (in the usual sense) of $\mathfrak{A}$, which is weak-* compact, but which contains a host of non-normal states.

Two pathological examples The following simple examples are useful as illustrations of the range of possibilities comprended by the formalism sketched above.

Example 2.10 (Two bits) Let $\mathcal{M}=\left\{\left\{a, a^{\prime}\right\},\left\{b, b^{\prime}\right\}\right\}$ be a test space consisting of two yes-no tests, one with outcomes $a$ and $a^{\prime}$, the other with outcomes $b$ and $b^{\prime}$. The space of all probability weights on $\mathcal{M}$

\footnotetext{
${ }^{3}$ This is one setting in which we would often not require $\Omega$ to be closed. For instance, if $\Omega=\Delta_{\sigma}(S, \Sigma)$, then $\Omega$ is not closed in the topology of event-wise convergence.

${ }^{4}$ Gleason's Theorem tells us that if $\operatorname{dim}(\mathcal{H})>2$, all probability weights arise in this way, but we will not make use of this fact.
} 
is essentially the unit square, since such a weight $\alpha$ is determined by the pair $(\alpha(a), \alpha(b))$. While the state space is not a simplex, pure states are dispersion-free. The square bit $B$ and diamond bit $B^{\prime}$ are the probabilistic models having the same test space, namely

$$
\mathcal{M}(B)=\mathcal{M}\left(B^{\prime}\right)=\mathcal{M},
$$

but the two different state spaces pictured below:

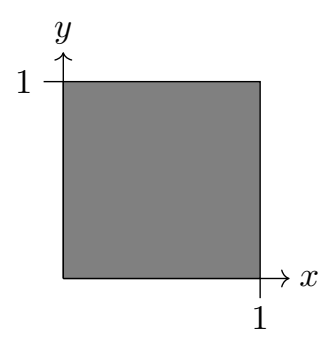

$\Omega(B)=$ all prob weights on $\mathcal{M}(B)$

Figure 1: two bits

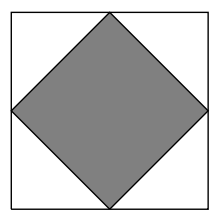

$\Omega\left(B^{\prime}\right)$

The square bit figures, directly or indirectly, in the large literature on "PR boxes" inaugurated by [22]. While the state space of $B^{\prime}$ is affinely isomorphic to that of $B$, it interacts with $\mathcal{M}$ differently. While both models are unital, in $B^{\prime}$, for each outcome $x$ there is a unique state $\delta_{x}$ with $\delta_{x}(x)=1$.

Example 2.11 (The Wright triangle ${ }^{5}$ ) A more interesting example consists of three overlapping, threeoutcome tests pasted together in a loop, as in Figure 2:

$$
\mathcal{M}=\{\{a, x, b\},\{b, y, c\},\{c, z, a\}\}
$$

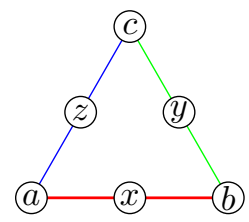

Figure 2: A "Greechie diagram" of the Wright Triangle, in which outcomes belonging to a commont test lie along a smooth curve (here, a straight line).

Here the space $\operatorname{Pr}(\mathcal{M})$ of all probability weights on $\mathcal{M}$ is three-dimensional, since such a weight is uniquely determined by the triple $(\alpha(a), \alpha(b), \alpha(c))$. It is not difficult to check that $\Omega$ is spanned by four dispersionfree states, determined by

$$
\alpha(a)=\alpha(y)=1, \beta(b)=\beta(z)=1, \gamma(c)=\gamma(x)=1 \text { and } \delta(x)=\delta(y)=\delta(y)=1,
$$

and a fifth, non-dispersion free pure state

$$
\epsilon(a)=\epsilon(b)=\epsilon(c)=1 / 2, \epsilon(x)=\epsilon(y)=\epsilon(z)=0 .
$$

In particular, $\operatorname{Pr}(\mathcal{M})$ is not a simplex, and not all pure states are dispersion-free. 


\subsection{Probabilistic models linearized}

It is often convenient to consider the vector space $\mathbf{V}(A) \leq \mathbb{R}^{X(A)}$ spanned by the state space $\Omega(A)$. This carries an obvious pointwise order. Writing $\mathbf{V}(A)_{+}$for the cone of all $\alpha \in \mathbf{V}(A)$ with $\alpha(x) \geq 0$ for all $x \in X(A)$, it is not difficult to see that each $\alpha \in \mathbf{V}(A)_{+}$has the form $\alpha=r \beta$ where $\beta \in \Omega(A)$ and $r \geq 0$. Moreover, the coefficient $r$ is unique, so $\Omega(A)$ is a base for the cone $\mathbf{V}(A)_{+}$.

An effect on $A$ is a functional $f: \mathbf{V}(A) \rightarrow \mathbb{R}$ with $0 \leq f(\alpha) \leq 1$ for $\alpha \in \Omega(A)$. Every outcome $x \in X(A)$ determines an effect $\widehat{x} \in \mathbf{V}(A)^{*}$ by evaluation, that is, by $\widehat{x}(\alpha)=\alpha(x)$ for all $\alpha \in \mathbf{V}(A)$. We can regard an arbitrary $f$ as representing an "in-principle" measurement outcome, not necessarily included among the outcomes in $X(A)$, but consistent with the convex structure of $\Omega(A): f(\alpha)$ is the probability of the effect $f$ occuring (if measured) in the state $\alpha$. There is a unique unit effect $u_{A}$ given by $u(\alpha)=1$ for all $\alpha \in \Omega(A)$, representing the trivial outcome "something happened". If $f$ is an effect, so is $u_{A}-f=: f^{\prime}$; we interpret $f^{\prime}$ as representing the event that $f$ did not occur. Thus, the pair $\left\{f, f^{\prime}\right\}$ is an in-principle test (measurement, experiment) not necessarily included in $\mathcal{M}(A)$. More generally, if $f_{1}, \ldots, f_{n}$ are distinct effects with $\sum_{i} f_{i}=u_{A}$, we regard $\left\{f_{1}, \ldots, f_{n}\right\}$ as an "in principle" measurement or experiment - the standard term is "discrete observable" - not necessarily included in the catalogue $\mathcal{M}(A)$, but consistent with the structure of $\Omega(A)$. Each test $E \in \mathcal{M}(A)$ gives rise to a discrete observable $\{\widehat{x} \mid x \in E\}$ in this sense.

Examples 2.12 (a) As an illustration, let $(S, \Sigma)$ be a measurable space, and let $\Delta=\Delta(S, \Sigma)$ be the space of finitely additive probability measures on $\Sigma$. Then $\mathbf{V}(\Delta)$ is the space of all signed measures $\mu=\mu_{+}-\mu_{-}$ on $\Sigma$, where $\mu_{+}$and $\mu_{-}$are positive measures. Any measurable function $\zeta: S \rightarrow[0,1]$ defines an effect $a$ through the recipe $a(\mu)=\int_{S} \zeta(s) d \mu(s)$. One can think of such an effect as a "fuzzy" or "unsharp" version of an indicator function (modelling, perhaps, a detector that responds with probability $\zeta(s)$ when the system is in a state $s$ ). The unit effect is the constant function 1. A discrete observable, accordingly, represents a generalization of a "fuzzy" or unsharp version of a finite measurable partition.

(b) In the case of a quantum-mechanical model, say $A=A(\mathcal{H})$, if we identify $\Omega(A)$ with the set of density operators on $\mathcal{H}$, then $\mathbf{V}(A)$ can be identified with the space of all compact self-adjoint operators on $\mathcal{H}$, ordered in the usual way; $\mathbf{V}(A)^{*}$ is then naturally isomorphic, as an ordered vector space, to the space of all bounded self-adjoint operators on $\mathcal{H}$, and an effect is an effect in the usual quantum-mechanical sense, that is, a positive self-adjoint operator $a$ with $0 \leq a \leq \mathbf{1}$, where $\mathbf{1}$ is the identity operator on $\mathcal{H}$, which serves as the unit effect in this case. A discrete observable corresponds to a discrete positive-operator valued measure (POVM) with values in $\mathbb{N}$.

Unlike a test in $\mathcal{M}(A)$, a general discrete observable on $A$ can have repeated values. Nevertheless, by considering their graphs, such observables can be organized into a test space, as follows. Here, $\left[0, u_{A}\right]$ denotes the set of all effects on $A$, and $\left(0, u_{A}\right]$ denotes the set of non-zero effects.

Definition 2.13 (Ordered tests) The space of ordered tests over the convex set $\Omega$ is the test space $\mathcal{M}^{o}(\Omega)$ with

(a) Outcome-space $X^{o}(\Omega):=\mathbb{N} \times(0, u]$;

(b) Tests, those finite sets $a \subseteq X^{o}$ that are graphs of mappings $a: I \rightarrow(0, u]$ with $\sum_{i \in I} a_{i}=u$, where $I \subseteq \mathbb{N}$ is finite.

In other words, tests in $\mathcal{M}^{o}(\Omega)$ are (graphs of) finite discrete observables with finite value-spaces $I \subseteq \mathbb{N}$. Every state $\alpha \in \Omega$ defines a probability weight $\alpha^{o}$ on $\mathcal{M}^{o}(\Omega)$ by $\alpha^{o}(i, a)=\alpha(a) .{ }^{6}$ In effect, $\mathcal{M}^{o}(\Omega)$ is the largest test space supporting $\Omega$ as a separating set of probability weights.

\footnotetext{
${ }^{6}$ It can be shown that, conversely, every probability weight on $\mathcal{M}^{o}(\Omega)$ is determined by a finitely-additive normalized measure on the effect algebra $[0, u]$, and hence, by a positive linear functional in $\mathbf{V}(A)^{* *}$. Hence, probability weights on $\mathcal{M}^{o}(\Omega)$ that are continuous on $(0, u]$ in the latter's relative weak-* topology, arise from elements of $\Omega$.
} 


\subsection{Probabilistic theories}

A probabilistic theory ought to be more than a collection of probabilistic models. One also needs a collection of allowed mappings between such models turning $\mathcal{C}$ into a category. There are many plausible ways to define a morphism between probabilistic models, some more restrictive, some very general (see, e.g., [4, 11]). For the purposes of this chapter, it will be sufficient to consider the following, relatively strict definition (due to Foulis and Randall [11]). Recall that $\mathcal{E} \boldsymbol{v}(\boldsymbol{\mathcal { M }})$ denotes the set of events (subsets of tests) for a test space $\mathcal{M}$. Given two events $a, b \in \mathcal{E} \boldsymbol{v}(\mathcal{M})$, we write $a \perp b$ to mean that $a \cap b=\emptyset$ and $a \cup b$ is again an event. That is, $a \perp b$ iff $a$ and $b$ are compatible (jointly testable), and mutually exclusive.

Definition 2.14 (Interpretations) Let $\mathcal{M}$ and $\mathcal{M}^{\prime}$ be test spaces with outcome-sets $X$ and $X^{\prime}$. An interpretation from $\mathcal{M}$ to $\mathcal{M}^{\prime}$ is a mapping $\phi: X \longrightarrow \mathcal{E} \boldsymbol{v}\left(\mathcal{M}^{\prime}\right)$ such that if $x \perp y$ in $X$, then $\phi(x) \perp \phi(y)$ in $\mathcal{E} \boldsymbol{v}\left(\mathcal{M}^{\prime}\right)$ and $\bigcup_{x \in E} \phi(x) \in \mathcal{M}^{\prime}$ for all $E \in \mathcal{M}$.

In other words, an interpretation $\phi: \mathcal{M} \rightarrow \mathcal{M}^{\prime}$ allows us to regard (to interpret!) each test $E \in \mathcal{M}$ as a coarse-grained version of a test in $\mathcal{M}^{\prime}$, in a non-contextual way.

Definition 2.14 (Continued): The following terminology will be useful. An interpretation $\phi: \mathcal{M} \rightarrow \mathcal{M}^{\prime}$ is outcome-preserving iff $\phi(x)$ is either empty or a singleton for every $x \in X$, in which case we can identify $\phi$ with the corresponding partial mapping from the outcome space $X=\bigcup \mathcal{M}$ to the outcome space $X^{\prime}=\bigcup \mathcal{M}^{\prime}$. If $\phi(x)$ is non-empty for every $x \in \bigcup \mathcal{M}$, then $\phi$ is positive. A positive, outcome-preserving interpretation $\phi: \mathcal{M} \rightarrow \mathcal{M}^{\prime}$, amounts to a mapping $\phi: X \rightarrow X^{\prime}$ with $\phi(\mathcal{M}) \subseteq \mathcal{M}^{\prime}$. Where this mapping $\phi$ is injective, allowing us to identify $\mathcal{M}$ with a subset of $\mathcal{M}^{\prime}$, we call it an embedding. Where $\phi$ is positive and $\phi(\mathcal{M})=\mathcal{M}^{\prime}$, we will say that $\phi$ is a cover of $\mathcal{M}^{\prime}$ by $\mathcal{M}$. Where $\phi$ is bijective on both outcomes and tests, we call it an isomorphism of test spaces.

Examples 2.15 (a) If $(S, \Sigma)$ and $(T, \Xi)$ are measuable spaces, any measurable mapping $f: S \rightarrow T$ gives rise to an outcome-preserving interpretation $\phi: \mathcal{D}(T, \Xi) \rightarrow \mathcal{D}(S, \Sigma)$, given by $\phi(b)=\left\{f^{-1}(b)\right\}$ when $f^{-1}(b) \neq \emptyset$, and $\phi(b)=\emptyset$ otherwise.

(b) Suppose $K_{1}$ and $K_{2}$ are compact convex sets and $f: K_{1} \rightarrow K_{2}$ is an affine mapping, we can define an outcome-preserving interpretation $\phi: \mathcal{M}^{o}\left(K_{2}\right) \rightarrow \mathcal{M}^{o}\left(K_{1}\right)$ by setting

$$
\phi(i, b)= \begin{cases}\{(i, b \circ f)\} & b \circ f \neq 0 \\ \emptyset & b \circ f=0\end{cases}
$$

(c) For an example of a positive but not outcome-preserving interpretation, consider the inclusion mapping $\mathcal{E} \boldsymbol{v}(\mathcal{M}) \backslash\{\emptyset\} \rightarrow \mathcal{E} \boldsymbol{v}(\mathcal{M})$ : we can understand this as an interpretation $\mathcal{M}^{\#} \rightarrow \mathcal{M}$ taking a non-empty event qua outcome of $\mathcal{M}^{\#}$ to the same event viewed as a set of outcomes of $\mathcal{M}$.

Notice that any interpretation $\phi: \mathcal{M}_{1} \rightarrow \mathcal{M}_{2}$ gives rise to an affine mapping $\phi^{*}: \operatorname{Pr}\left(\mathcal{M}_{2}\right) \rightarrow \operatorname{Pr}\left(\mathcal{M}_{1}\right)$, defined for $\beta \in \Omega\left(\mathcal{M}_{2}\right)$ by $\phi^{*}(\beta)=\beta \circ \phi$.

Definition 2.16 (Interpretations of models) An interpretation $\phi: A \rightarrow B$ from a probabilistic model $A$ to a probabilistic model $B$ is an interpretation $\phi: \mathcal{M}(A) \rightarrow \mathcal{M}(B)$ such that $\phi^{*}(\Omega(B)) \subseteq \Omega(A)$. We say that $\phi$ is

(a) an embedding iff $\phi$ is an embedding of test spaces and $\phi^{*}$ is surjective;

(b) a cover iff $\phi$ is a cover of $\mathcal{M}(B)$ by $\mathcal{M}(A)$, in which case $\phi^{*}$ is injective.

The affine mapping $\phi^{*}: \Omega(B) \rightarrow \Omega(A)$ extends uniquely to a positive linear mapping $\phi^{*}: \mathbf{V}(B) \rightarrow \mathbf{V}(A)$, satisfying $u_{A} \circ \phi^{*}=u_{B}$. An apparently much more general notion of a morphism from a model $A$ to 
a model $B$ is a positive linear mapping $\Phi: \mathbf{V}(B) \rightarrow \mathbf{V}(A)$ with the feature that $u_{A} \circ \Phi \leq u_{B}$, that is, $u_{B}(\Phi(\beta)) \leq 1$ for all $\beta \in \Omega(B)$ [4]. Such a map represents a possibly "lossy" process that, given as input a state $\beta \in \Omega(B)$, produces an output state $\alpha:=\Phi(\beta) / u_{B}(\Phi(\beta))$ with probability $u_{B}(\Phi(\beta))$ - or, if $u_{B}(\Phi(\beta))=0$, simply destroys the system. However, given such a mapping $\Phi$, and letting $\nabla(A)$ denote the convex set of sub-normalized states $r \alpha$ were $r \in[0,1]$ and $\beta \in \Omega(B)$, we can define an interpretation $\phi: \mathcal{M}^{o}(\nabla(A)) \rightarrow \mathcal{M}^{o}(\Omega(B))$ as in Example 2.15 (b) above. Thus, we actually lose very little, if any, generality in restricting attension to categories of probabilistic models and interpretations. In the remainder of this chapter, a probabilistic theory will always mean such a category.

\section{Classicality and Classical Representations}

There are various ways of representing probabilistic models in terms of classical probabilistic models, most of them reasonably familiar in the context of QM. In this section, I want to discuss two of these that are rather canonical, both in the informal sense that they are more or less obvious, and apply to more or less arbitrary probabilistic models, and also in the technical sense that the constructions involved are actually functorial. Of these, it is my sense that only the first is really well known.

\subsection{Classical models and classical embeddings}

Before we can discuss these representations, we need to address head on the central concern of this chapter: what does it mean (what ought it to mean) for a probabilistic model $A$ to be "classical"? Whatever our final answer, presumably we would at least wish to consider models $A(S, \Sigma):=\left(\mathcal{D}(S, \Sigma), \Delta_{\sigma}(S, \Sigma)\right)$ as classical. However, as discussed earlier, even classically it won't do to restrict attention to such models: any model of the form $(\mathcal{D}(S, \Sigma), \Omega)$ where $\Omega$ is any subset of $\Delta_{\sigma}(S, \Sigma)$, should almost certainly count as "classical", in the sense of falling within the scope of classical probability theory [18].

We should also pause to ask whether or not the restriction to $\sigma$-additive measures should be regarded as one of the defining features of classicality. If $\mu$ is a finitely additive measure on an algebra $\Sigma$ of sets, then letting $T=\beta(\Sigma)$ be the Stone space of $\Sigma$, regarded as a Boolean algebra, one finds that, owing to the fact that elements of $\Sigma$ are clopen in $T$ and $T$ is compact, no countable disjoint family of sets in $\Sigma$ has union equal to $T$. Hence, every measure on $(T, \Sigma)$ is countably additive by default. In different language, whether or not a measure on a Boolean algebra $\Sigma$ is countably additive depends on the particular representation of $\Sigma$ as an algebra of sets.

For this reason, it seems prudent to allow any Kolmogorovian model, in the sense of Example 2.8 - that is, any probabilistic model of the form $(\mathcal{D}(S, \Sigma), \Omega)$, where $\Sigma$ is any algebra of subsets of $S$ and $\Omega$ is any convex set of probability measures on $\Sigma$, countably additive or otherwise, to count as "classical". But if this is so, it seems equally reasonable to admit as classical models of the form $(\mathcal{M}, \Omega)$ as long as $\mathcal{M} \subseteq \mathcal{D}(S, \Sigma)$ and $\Omega \subseteq \Delta(S, \Sigma)$. That is, models admitting embeddings, in the sense of Definition 2.16, into Kolmogorovian models in the sense of Example 2.8, should themselves be considered "classical" in a broad sense.

When does an abstract model $(\mathcal{M}, \Omega)$ admit such a classical embedding? If $\phi: \mathcal{M}(A) \rightarrow \mathcal{D}(S, \Sigma)$ is an interpretation, then every point-mass $\delta_{s}$ in $\Delta(S, \Sigma)$ pulls back along $\phi$ to a dispersion-free probability weight on $\mathcal{M}(A)$. If $\phi$ is to be an embedding of test spaces, there must be enough of these dispersion-free states to separate outcomes in $X(A)$. Moreover, if $\phi$ is to be an embedding of probabilistic models, every state $\alpha \in \Omega(A)$ must belong to the closed convex hull of these dispersion-free weights.

Definition 3.1 (UDF models) A probabilistic model $A$ is unitally dispersion-free or UDF iff every state $\alpha \in \Omega(A)$ lies in the closed convex hull of the set of dispersion-free probability weights on $\mathcal{M}(A)$. 
Versions of the following can be found in many places in the quantum-logical literature.

Lemma 3.2 A model admits a classical embedding iff it is UDF.

Proof: The "only if" direction is clear from the discussion above. For the converse, suppose $(\mathcal{M}, \Omega)$ is $\mathrm{UDF}$, and let $S$ be the set of dispersion-free probability weights on $\mathcal{M}$. For each $x \in X:=\bigcup \mathcal{M}$, let

$$
a_{x}:=\{s \in S \mid s(x)=1\} \subseteq S .
$$

Then $\left\{a_{x} \mid x \in E\right\}$ is a partition of $S$. Let $\Sigma$ be the algebra of subsets of $S$ generated by sets of the form $a_{x}$. Then the mapping $\phi: X \rightarrow \Sigma$ given by $\phi(x)=a_{x}$ gives rise to an outcome-preserving interpretation $\mathcal{M} \rightarrow \mathcal{D}(S, \Sigma)$, which is an embedding if and only if $S$ separates points of $X$. The mapping $\phi^{*}: \mathbb{R}^{\Sigma} \rightarrow \mathbb{R}^{X}$ given by $\phi^{*}(\mu)(x)=\mu\left(a_{x}\right)$ is continuous with respect to the product topologies on these spaces, and for each $s \in S, \phi^{*}\left(\delta_{s}\right)=s$. It follows that $\overline{\operatorname{con}}(S)=\phi^{*}(\Delta(S, \Sigma))$. Hence, the probability weights in $\overline{\operatorname{con}}(S)$ have a common classical explanation. Given any closed convex set $\Omega \subseteq \overline{\operatorname{con}}(S)$, we then have an embedding of the model $(\mathcal{M}, \Omega)$ into the Kolmogorovian model $\left(\mathcal{D}(S, \Sigma),\left(\phi^{*}\right)^{-1}(\Omega)\right)$.

Let me stress that it is not sufficient for $\mathcal{M}(A)$ simply to have a large supply of dispersion-free probability weights: we also require that all of the allowed states $\alpha \in \Omega(A)$ arise as (limits of) averages of these. As an example, the Wright Triangle admits many dispersion-free states, but also a non-dispersion-free extreme state, which can not be explained in terms of a classical embedding.

Of course, if $\mathcal{M}(A)$ has no dispersion-free states at all, then such a classical embedding is impossible in any case. Gleason's Theorem tells us that $\Omega(\mathcal{H})$ has no dispersion-free states if $\operatorname{dim}(\mathcal{H}) \geq 3$; this is the substance of one of the strongest "no-go" theorems for hidden variables. However, this is far from the end of the story. For one thing, the classical embeddings considered above are non-contextual. That is, if $x \in E \cap F$, where $E, F \in \mathcal{M}(A)$, then $\phi(x)$ does not depend on whether we regard $x$ as an outcome of $E$ or of $F$. As was realized very early on, making $\phi(x)$ depend also on the choice of "measurement context" (e.g., $E$ or $F$, above) allows for much more flexibility.

Besides the existence of an abundance of dispersion-free states, a distinctive feature of Kolmogorovian models is that any two partitions $E, F \in \mathcal{D}(S, \Sigma)$ can be regarded as coarse-grained versions of a common refinement: if

$$
G=\{a \cap b \mid a \in E, b \in F, a \cap b \neq \emptyset\}
$$

then $G \in \mathcal{D}(S, \Sigma)$, and every $a \in E$, and likewise every $b \in F$, is in an obvious sense equivalent to a subset of $G$. This allows us to make a simultaneous joint measurement of $E$ and $F$, by performing a measurement of $G$. One can define a general notion of the refinement of one test by another in the context of an arbitrary probabilistic model, as follows:

Definition 3.3 (Refinement) A test $E \in \mathcal{M}(A)$ refines a test $F \in \mathcal{M}(A)$ iff there exists a surjection $f: E \rightarrow F$ such that $\alpha(y)=\alpha\left(f^{-1}(y)\right)$ for every $y \in F$ and every state $\alpha \in \Omega(A)$.

Recall that a probabilistic model $A$ is unital if every outcome $x \in X(A)$ has probability one in at least one state, i.e., there is at least one state $\alpha \in \Omega(A)$ with $\alpha(x)=1$.

Theorem 3.4 Let A be a unital model in which every pair of tests has a common refinement. Then A has a classical embedding.

The proof is given in Appendix A.

Conversely, of course, if $A$ has a classical embedding, then $\mathcal{M}(A)$ embeds in a test space $\mathcal{D}(S, \Sigma)$, in which every pair of tests has a common refinement. Thus, a necessary and sufficient condition for a unital model $A$ to have a classical embedding is that it be possible to embed $A$ in a model $B$ in which every pair of tests 
has a common refinement. To this extent, one might reasonably say that it is the existence (or at least, the mathematical possibility) of joint measurements that is the hallmark of "classical" probabilistic theories. However, it is very important to note again at this point, that this is an entirely contingent feature of classical probability theory. That is, there is no "law of thought" that tells us that any two measurements or experiments can be performed jointly.

\subsection{Classical Extensions}

An embedding is not the only way of explaining one mathematical object in terms of another. One can also consider representing the object to be explained as a quotient of a more familiar object. In connection with probabilistic models, this line of thinking was explored by Holevo [15] and, slightly later, by Bugajsky and Beltrametti ([6]; see also $[8,14]$.) In the literature, this sort of representation of a quantum model is usually called a classical extension. The basic idea is that any convex set can arise as a quotient of another under an affine surjection. As a simple example, a square can arise as the projection of a regular tetrahedron (a 4-simplex) on a plane.

To develop this idea further, we will need a bit of background on Choquet theory [1]. Recall that the $\sigma$-field of Baire sets of a compact Hausdorff space $S$ is the smallest $\sigma$-algebra containing all sets of the form $f^{-1}(0)$ where $f$ ranges over continuous real-valued functions on $S$. Let $K$ be a compact convex set, that is, a compact, convex subset of a locally convex topological vector space $V$, and let $\Delta_{o}(K)$ be the set of Baire probability measures thereon. This is an infinite-dimensional simplex in the sense that $\mathbf{V}\left(\Delta_{o}(K)\right)$ is a vector lattice [1]. For each $\mu \in \Delta(K)$, define the barycenter of $\mu, \widehat{\mu} \in V^{* *}$, by

$$
\widehat{\mu}(f)=\int_{K} f d \mu
$$

for all functionals $f \in V^{*}$. Using the Hahn-Banach Theorem, one can show that $\widehat{\mu} \in K$ ([1], I.2.1). Thus, we have an affine mapping $\mu \mapsto \widehat{\mu}$ from $\Delta_{o}(K)$ to $K$. The barycenter of the point-mass $\delta_{\alpha}$ at $\alpha \in K$ is evidently $\alpha$, so this mapping is surjective. In this sense, every compact convex set is the image of a simplex under an affine mapping.

Definition 3.5 (Classical extensions) A classical extension of a probabilistic model $A$ consists of a measurable space $(S, \Sigma)$ and an affine surjection $q: \Delta_{\sigma}(S, \Sigma) \rightarrow \Omega(A)$.

This makes no reference to $\mathcal{M}(A)$. However, the affine surjection $q: \Delta_{o}(S) \rightarrow \Omega(A)$ can be dualized to yield a mapping $q^{*}: X(A) \rightarrow \operatorname{Aff}\left(\Delta_{o}(S)\right)$ taking every outcome $x \in X(A)$ to the functional $q^{*}(x)(\mu)=\widehat{\mu}(x)$, which is evidently an effect on $\Delta(S)$. Now, such an effect can be understood as a "unsharp" version of an indicator function, as discussed in Example 2.12(a) Accordingly, since $\sum_{x \in E} q^{*}(x)$ is identically 1, $q^{*}$ is an effect-valued weight on $\mathcal{M}(A)$, representing each test as a partition of 1 by such fuzzy indicator functions, that is, $q^{*}(E)$ is a (discrete) "unsharp random variable" on $S$. For more on this, see [2]. In such a representation, in other words, the state space is essentially classical (it's simply a set of probability measures), while outcomes and tests become "unsharp". While this may represent a slight extension of the apparatus of standard, Kolmogorovian probability theory, it is certainly within the scope of classical probability theory in the somewhat wider sense that concerns us here.

Another way to put all this is that a classical extension comes along with (and determines) an interpretation $\mathcal{M}(A) \rightarrow \mathcal{M}^{o}(\Delta(S, \Sigma))$, where the latter is the space of ordered tests on $\Delta(S, \Sigma)$, in the sense of Definition 2.13. This then gives us an interpretation of models $A \rightarrow\left(\mathcal{M}^{o}(\Delta(S, \Sigma)), \Delta(S, \Sigma)\right)$. In this sense, a classical extension is an "unsharp" version of a classical embedding. However, as the discusssion above shows, every probabilistic model has a canonical classical extension obtained by taking $S=\Omega(A)$ and $\Sigma$ the Baire field of $\Omega$. This construction is even functorial, since the construction $A \mapsto \Omega(A)$ is (the object part of) a contravariant functor from the category of probabilistic models to the category of compact convex sets, while $K \mapsto \Delta_{0}(K)$ and $K \mapsto \mathcal{M}^{o}(K)$ are, respectively, a covariant endofunctor on the category of 
compact convex sets and continuous affine maps, and from this category to the category of test spaces and interpretations. Putting these together gives us a covariant functor $A \mapsto\left(\mathcal{M}^{o}\left(\Delta_{0}(\Omega(A))\right), \Delta_{0}(\Omega(A))\right)$ from arbitrary (locally finite) probabilistic models to unsharp Kolmogorovian models.

One can also construct a classical extension in which the carrier space is the set $K_{\text {ext }}$ of extreme points of $K$. This need not be a Borel subset of $K$; however, it becomes a measurable space if we let $\Sigma_{\text {ext }}$ be the trace of $K$ 's Baire field $\Sigma_{o}$ on $K_{\text {ext }}$, i.e., $\Sigma_{\text {ext }}=\left\{b \cap K_{\text {ext }} \mid b \in \Sigma\right\}$. If $\mu$ is a probability measure on $\Sigma_{\text {ext }}$, then we can pull this back along the boolean homomorphism $\phi: b \mapsto b \cap K_{\text {ext }}$ to a probability measure $\mu \circ \phi$ on $\Sigma_{o}$, the barycenter of which, as defined above, defines a barycenter for $\mu$; that is, we take $\widehat{\mu}:=\widehat{\mu \circ \phi}$. The Bishop-deLeeuw Theorem (see [1], I.4.14) asserts that every point of $K$ can be represented as the barycenter of a $\sigma$-additive probability measure on $\Sigma_{\text {ext }}$.

We can regard probability measures $\mu$ in the simplex $\Delta(S), S=\Omega(A)_{\text {ext }}$, as representing ensembles of pure states, that is, preparation procedures that produce a particular range of pure states, with prescribed probabilities. The quotient map $\Delta(S) \rightarrow \Omega(A)$ simply takes each such ensemble to its probability-weighted average. Where $\Omega(A)$ is not a simplex, many different ensembles will yield this same average; operationally, that is, using the measurements available in $\mathcal{M}(A)$, distinct ensembles averaging to the same state are indistinguishable, so treating a state $\alpha \in \Omega(A)$ as "really" arising from one, rather than another, such ensemble represents a kind of contextuality, what Spekkens [23] calls preparation contextuality.

\subsection{Semiclassical Covers}

Recall from Section 2.1 that the semiclassical cover, $\widetilde{\mathcal{M}}$, of a test space $\mathcal{M}$ is given by

$$
\widetilde{\mathcal{M}}=\{\widetilde{E} \mid E \in \mathcal{M}\},
$$

where, for $E \in \mathcal{M}, \widetilde{E}=\{(x, E) \mid x \in E\}$. The outcome-set of $\widetilde{\mathcal{M}}$ is thus $\widetilde{X}=\{(x, E) \mid x \in E \in \mathcal{M}\}$, i.e., the coproduct of the tests $E \in \mathcal{M}$. There is an obvious outcome-preserving interpretation $\pi: \widetilde{\mathcal{M}} \rightarrow \mathcal{M}$, given by $\pi(x, E)=\{x\}$. Any probability weight $\alpha$ on $\mathcal{M}$ pulls back along this to a probability weight $\widetilde{\alpha}:=\pi^{*}(\alpha)$ on $\widetilde{\mathcal{M}}$, given by $\widetilde{\alpha}(x, E):=\alpha(x)$ for all $x \in E \in \mathcal{M}(A)$. Given a model $A$, we define the semiclassical cover of $A$ to be the model $\widetilde{A}:=(\widetilde{\mathcal{M}}(A), \widetilde{\Omega}(A)\}$, where $\widetilde{\mathcal{M}}(A)$ is the semiclassical cover of $\mathcal{M}(A)$ and $\widetilde{\Omega}(A):=\{\widetilde{\alpha} \mid \alpha \in \Omega\}$. The mapping $\alpha \mapsto \widetilde{\alpha}$ is an affine injection, so $\widetilde{\Omega}(A)$ is isomorphic to $\Omega(A)$. Thus, $\widetilde{A}$ differs from $A$ only in the structure (or the comparative lack of structure) of its test space.

Since $\widetilde{\mathcal{M}}(A)$ is semiclassical, its space $\operatorname{Pr}(\widetilde{\mathcal{M}})$ of probability weights is essentially the Cartesian product $\Pi_{E \in \mathcal{M}} \Delta(E)$ of the finite-dimensional simplices $\Delta(E)$ of probability weights on the various tests $E \in \mathcal{M}$. Indeed, we can represent $\alpha \in \operatorname{Pr}(\widetilde{\mathcal{M}(A)})$ uniquely as $\left(\alpha_{E}\right)$ where $\alpha_{E}$ is $\alpha$ 's restriction to $E \in \mathcal{M}(A)$. Hence, $\widetilde{\mathcal{M}}$ supports a wealth of dispersion-free probability weights, namely, those weights obtained by selecting a dispersion-free probability weight — a point-mass — from each simplex $\Delta(E)$.

In what follows, I'll write $S(A)$ for the set of all such dispersion-free states on $\widetilde{\mathcal{M}}(A)$. It is easy to show that these are exactly the extreme probability weights on $\widetilde{\mathcal{M}}(A)$, and that $S(A)$ is closed in $\operatorname{Pr}(\widetilde{\mathcal{M}}(A))$ (see Appendix B). This allows us to represent every $\alpha \in \operatorname{Pr}(\widetilde{\mathcal{M}})$ as the barycenter of a Borel probability measure on $S(A)$ (as every Baire probability measure on a compact space has a unique Borel extension). Letting $\Delta(S(A))$ denote the simplex of Borel probability measures on $S(A)$, this gives us an affine surjection $\phi: \Delta(S(A)) \rightarrow \operatorname{Pr}(\widetilde{\mathcal{M}}(A))$. Also, $\psi:(x, E) \mapsto\{\alpha \in S \mid \alpha(x, E)=1\}$ gives an interpretation of each test $\widetilde{E} \in \widetilde{\mathcal{M}}(A)$ as an element of $\mathcal{D}(S, \Sigma)$, where $\Sigma$ is the Borel algebra of $S(A)$, and it is straightforward that $\phi=\psi^{*}$. We therefore have the following picture: 


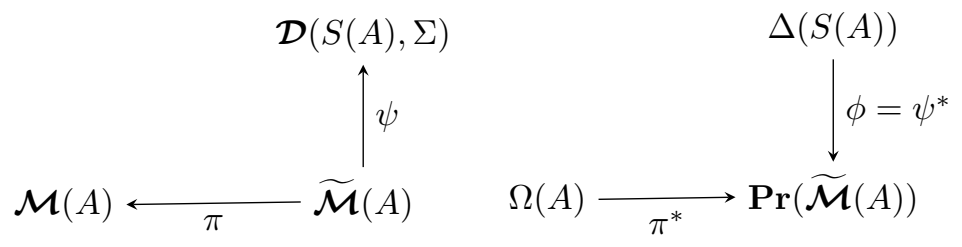

The dual mapping $\pi^{*}(\alpha)(x, E)=\alpha(x)$ is injective, while $\psi^{*}$ is surjective: again, $\Omega(A)$ is a convex subset of a quotient of a simplex. However, in this representation observables associated with tests $E \in \mathcal{M}(A)$ correspond to sharp classical random variables on $S(A)$ : letting $\widetilde{a}_{x}=\{s \in S(A) \mid s(x, E)=1\},\left\{a_{x} \mid x \in E\right\}$ is a partition of $S(\widetilde{A})$.

This gives us a very simple representation of an arbitrary model as a quotient of a sub-model of Kolmogorovian model. To the extent that a representation of a model $A$ as a quotient of a model $B$ allows us to "explain" the model $A$ in terms of the model $B$, and to the extent that representing a model $B$ as a submodel of a model $C$ also allows us to explain $B$ in terms of $C$, then - to the extent that "explains" is transitive - we see that every model can be explained in terms of a classical one. This construction is even functorial: if $\phi: A \rightarrow B$ is an interpretation from a model $A$ to a model $B$, there is a canonical extension of $\phi$ to an interpretation $\widetilde{\phi}: \widetilde{A} \rightarrow \widetilde{B}$, given by $\phi(x, E)=(\phi(x), \phi(E))$. Hence, an entire probabilistic theory can be rendered essentially classical, if we are willing to embrace contextuality.

Hidden Variables This is as good a place as any to clarify how the foregoing discussion connects to traditional notions of "hidden varibles" (or "ontological representations" [23]). Briefly, a (not necessarily deterministic, not necessarily contextual) hidden variables representation of a probabilistic model $A$ consists of a measurable space $\Lambda$ and, for every test $E \in \mathcal{M}$, a conditional probability distribution $p(\cdot \mid \lambda, E)$ on $E$ - that is, $p(x \mid \lambda, E)$ is a non-negative real number for each $x \in E$, and $\sum_{x \in E} p(x \mid \lambda, E)=1$. It is also required that, for every state $\alpha \in \Omega(A)$, there exist a probability measure $\mu$ on $\Lambda$ such that for every $x \in X(A)$ and every test $E$ with $x \in E$,

$$
\int_{\Lambda} p(x \mid \lambda, E) d \mu(\lambda)=\alpha(x) .
$$

Now, if we rewrite $p(x \mid \lambda, E)$ as $p_{\lambda}(x, E)$, we see that $p_{\lambda}$ is simply a probability weight on $\widetilde{\mathcal{M}}(A)$. We can then write (1) as

$$
\alpha(x)=\left(\int_{\Lambda} p_{\lambda} d \mu(\lambda)\right)(x, E)
$$

which merely asserts that $\alpha$ is the $\mu$-weighted average of a family of probability weights on $\widetilde{\mathcal{M}}(A)$, parametrized by $\Lambda$.

\subsection{Discussion}

In view of the classical representations discussed in sections 3.2 and 3.3 , it seems that we can always understand a "generalized probabilistic model", or, indeed, a generalized probababilistic theory, in terms of a classical model or theory. The only departure from classical Kolmogorovian probability theory, in these representations, is a restriction - which one can regard as epistemic or as "physical", as the case warrants - on which ensembles of probability measures one can prepare, which (classical) measurements one can perform, and hence, on which measurement outcomes and which mixtures of states one can distinguish. To be sure, we are under no obligation to take ensembles in $\Delta\left(\Omega_{\text {ext }}\right)$ or dispersion-free states on $\widetilde{\mathcal{M}}$ seriously as part of any given physical theory, and we may also prefer to do without them for reasons of mathematical economy; but both are mathematically meaningful, and certainly have a place in any developed general probability theory. To this extent, arbitrary probabilistic models, and even probabilistic theories, have fairly canonical "explanations" in Kolmogorovian terms. 
In the next section I will go just a step further and point out that, for models with certain strong symmetry properties (including quantum models), there is available a rather different kind of "classical" representation: one in which the model's probabilistic apparatus is entirely classical - there is, in fact, only one basic measurement. In such a representation it is, rather, the interplay between a system's symmetries, its states, and this perfectly classical probabilistic apparatus, that is (in a sense I try to pin down) perhaps not quite "classical".

\section{Dynamical Models and Dynamical States}

One of the things that most conspicuously distinguishes quantum probabilistic models from more general ones is the their very high degree of symmetry: given any two pure quantum states, there is a unitary operator taking one to the other, and likewise, given any two projective orthonormal bases, there is a unitary operator taking one to the other. In this section, I want to sketch a different kind of classical representation, available for locally finite models whenever such a great deal of symmetry is in play. This is mathematially similar to the representation in terms of the semiclassical cover; conceptually, however, it is leaner, in that it privileges a single observable, and encodes the structure of the model in the ways in which probabilities on this observable can change.

\subsection{Models with Symmetry}

In this section, we will consider probabilistic models $A$ that, in addition to the test space $\mathcal{M}(A)$ and state space $\Omega(A)$, are equipped with a distinguished symmetry group $G(A)$ acting on $X(A), \mathcal{M}(A)$ and $\Omega(A)$. In most applications, $G(A)$ will be a lie group and, in our finite dimensional setting, compact.

Definition 4.1 (Symmetries of test spaces) a symmetry of a test space $\mathcal{M}$ with outcome-set $X=$ $\bigcup \mathcal{M}$ is an isomorphism $g: \mathcal{M} \rightarrow \mathcal{M}$, or, equivalently, a bijection $g: X \rightarrow X$ such that $\forall E \subseteq X$, $g(E) \in \mathcal{M}$ iff $E \in \mathcal{M}$. We write $\operatorname{Aut}(\mathcal{M})$ for the group of all symmetries of $\mathcal{M}$ under composition.

Note that $\operatorname{Aut}(\mathcal{M})$ also has a natural right action on $\operatorname{Pr}(\mathcal{M})$, since if $g \in \operatorname{Aut}(\mathcal{M})$ and $\alpha \in \operatorname{Pr}(\mathcal{M})$, then $\alpha \circ g$ is again a probability weight, and the mapping $g^{*}: \alpha \mapsto \alpha \circ g$ is an affine bijection $\operatorname{Pr}(\mathcal{M}) \rightarrow \operatorname{Pr}(\mathcal{M})$.

Definition 4.2 (Dynamical models) A dynamical probabilistic model is a probabilistic model $A$ with a distinguished dynamical group $G(A) \leq \operatorname{Aut}(\mathcal{M}(A))$ under which $\Omega(A)$ is invariant [4].

I use the terms dynamical model and dynamical group because in situations in which $G(A)$ is a Lie group, a reversible dynamics for (or consistent with) the model will be a choice of a continuous one-parameter group $g \in \operatorname{Hom}(\mathbb{R}, G(A))$, that is, a mapping $t \mapsto g_{t}$ with $g_{t+s}=g_{t} g_{s}$, which we interpret at tracking the system's evolution over time: if $\alpha$ is the state at some initial time, $g_{t}(\alpha)=\alpha \circ g_{-t}$ is the state after the elapse of $t$ units of time. That $g$ is a homomorphism encodes a Markovian assumption about the dynamics, namely, that a system's later state depends only on its initial state and the amount of time that has passed, rather than on the system's entire history.

Definition 4.3 (Symmetric and fully symmetric models) A dynamical probabilistic model $A$ is symmetric iff $G(A)$ acts transitively on the set $\mathcal{M}(A)$ of tests, and the stabilizer, $G(A)_{E}$, of a (hence, any) test $E \in \mathcal{M}$ acts transitively on $E$. Note that this implies that all tests have a common size. We say that $A$ is fully symmetric iff, additionally, for any test $E \in \mathcal{M}(A)$ and any permutation $\sigma: E \rightarrow E$, there exists at least one $g \in G(A)$ with $g x=\sigma(x)$ for every $x \in E$. 
An equivalent way to express full symmetry is to say that all tests $E, F \in \mathcal{M}(A)$ have the same cardinality, and, for every bijection $f: E \rightarrow F$, there exists some $g \in G(A)$ with $g x=f(x)$ for every $x \in E$.

The quantum probabilistic model $A(\mathcal{H})$ is fully symmetric under the unitary group $\mathcal{U}(\mathcal{H})$, since any bijection between two (projective) orthonormal bases for $\mathcal{H}$ extends to a unitary operator.

We can reconstruct a symmetric test space $\mathcal{M}(A)$ from any one of its tests, plus information about its symmetries [27]. Suppose we are given, in addition to the group $G(A)$, a single test $E \in \mathcal{M}(A)$, an outcome $x_{o} \in E$, and the two subgroups

$$
H:=\{g \in G(A) \mid g E=E\} \text { and } K:=\left\{g \in G(A) \mid g x_{o}=x_{o}\right\} .
$$

We then have a canonical bijection $G(A) / K \rightarrow X(A)$, sending $g K$ to $g x_{o}$, and sending $\{h K \mid h \in H\}$ bijectively onto $E ; \mathcal{M}(A)$ can then be recovered as the orbit of $E$ in $X(A)$. Thus, more abstractly, one could start with a triple $(G, H, K)$ where $G$ is a group and $H, K$ are subgroups of $G$, define $X:=G / K$ and $E=\{h K \mid h \in H\}$, and set $\mathcal{M}=\{g E \mid g \in G\}$. If $H$ acts as the full permutation group on $E, \mathcal{M}$ will be fully symmetric under $G$. The choice of a closed convex set $\Omega \subseteq \operatorname{Pr}(\mathcal{M})$, invariant under $G$, completes the picture and gives us a $G$-symmmetric probabilistic model.

The simplest cases are those in which $H$ is a subgroup of $\mathfrak{S}(E)$, the group of permutations of $E$. This holds for ordinary quantum test spaces, since every permutation of an orthonormal basis determines a unique unitary. In what follows, we concentrate on this case. (For a projective quantum test space, in which $E$ is a maximal set of pairwise orthogonal rank-one projections, $H$ is generated by permutation matrices acting on $E$, plus unitaries commuting with these projections.)

\subsection{A Representation in terms of dynamical states}

We now reformulate these ideas in a way that gives more prominence to the chosen test $E \in \mathcal{M}(A)$. In brief: if we hold the test $E$ fixed, but retain control over both the state space $\Omega(A)$ and the dynamical group $G(A)$, we obtain a mathematically equivalent picture of the model, but one in which the probabilistic structure is, from a certain point of view, essentially classical.

Consider the following situation: one has some laboratory apparatus, defining an experiment with outcomeset $E$. This is somehow coupled to a physical system having a state-space $\Omega$, governed by a Lie group $G$. That is, $G$ acts on $\Omega$ in such a way that all possible evolutions of this system are described by an initial state $\alpha_{o} \in \Omega$ and a one-parameter subgroup $\left(g_{t}\right)_{t \in \mathbb{R}}$ of $G$. It is harmless, and will be convenient, to take $\Omega$ to be a right $G$-space, that is, to denote the image of state $\alpha \in \Omega$ under the action of $g \in G$ by $\alpha g$. We may suppose that the way in which the apparatus is coupled with the system manifests itself probabilistically, by a function

$$
p: \Omega \times E \rightarrow[0,1]
$$

giving the probability $p(\alpha, x)$ to obtain outcome $x \in E$ when the system is in state $\alpha{ }^{7}$ I will assume in what follows that the probability weights $p(\alpha, \cdot)$ separate outcomes in $E$, that is, that for all outcomes $x, y \in E$,

$$
(\forall \alpha \in \Omega p(\alpha, x)=p(\alpha, y)) \Rightarrow x=y \text {. }
$$

(If not, we can factor out the obvious equivalence relation on $E$.) I will also suppose that the experiment $E$ and the group $G$ together separate states, that is, knowing how the probabilities $p(\alpha g, x)$ vary with $g \in G$, for all outcomes $x \in E$, is enough to determine the state $\alpha$ uniquely. In other words,

$$
(\forall g \in G, \forall x \in E p(\alpha g, x)=p(\beta g, x)) \Rightarrow \alpha=\beta
$$

\footnotetext{
${ }^{7}$ I prefer to write this as $p(\alpha, x)$ rather than as $p(x \mid \alpha)$ in order to make the covariance conditions below come out more prettily.
} 
Notice that for each state $\alpha \in \Omega$, we can define a mapping $\widehat{\alpha}: G \rightarrow \Delta(E)$, where $\Delta(E)$ is the simplex of probability weights on $E$, given by

$$
\widehat{\alpha}(g)(x)=p(\alpha g, x) .
$$

Alternatively, we can treat $\widehat{\alpha}$ as a mapping $G \times E \rightarrow[0,1]$ with $\sum_{x \in E} \widehat{\alpha}(g, x)=1$, by writing $\widehat{\alpha}(g, x):=$ $\widehat{\alpha}(g)(x)$. I will leave it to context to determine which of these representations is intended. In either case, I will refer to $\widehat{\alpha}$ as the dynamical state associated with state $\alpha \in \Omega$. The state-separation condition (4) tells us that $\alpha \mapsto \widehat{\alpha} \in \Delta(E)^{G}$ is injective, so if we wish, we can identify states with the corresponding dynamical states.

The mapping $\widehat{\alpha} \in \Delta(E)^{G}$ is a (discrete) random probability measure on $G$, while $\widehat{\alpha}: G \times E \rightarrow[0,1]$ is a discrete Markov kernel on $G \times E$. Thus, nothing we have done so far takes us outside the range of classical probability theory. In fact, if $G$ is compact, that there is a natural measure on $G \times E$, namely the product of the normalized Haar measure on $G$ and the counting measure on $E$, such that

$$
\int_{G \times E} \widehat{\alpha}(g, x) d(g, x)=\int_{G}\left(\sum_{x \in E} \widehat{\alpha}(g, x)\right) d g=\int_{G} 1 d g=1 .
$$

In other words, for each state $\alpha \in \Omega, \widehat{\alpha}(g, x)$ defines a probability density on $G \times E$. Moreover, as $\sum_{x \in E} \widehat{\alpha}(g, x)=1$, the conditional density $\widehat{\alpha}(x \mid g)$ is exactly $\widehat{\alpha}(g) \in \Delta(E)$. Nevertheless, any symmetric probabilistic model provides an example of this scenario: simply chose a test $E \in \mathcal{M}(A)$, and let $p(\alpha, x)=$ $\alpha(x)$ and $\widehat{\alpha}(g)(x)=\alpha(g x)$ for all $\alpha \in \Omega(A)$ and any $x \in E$. Conversely, as we will see, one can reconstruct a symmetric probabilistic model $A$ with $E \in \mathcal{M}(A)$ and $\Omega \simeq \Omega(A)$ from the "classical" data described above, that is, the test $E$, the state space $\Omega$, the symmetry group $G$ and the probabilistic coupling $p$.

Definition 4.4 (Implementing a permutation) Let $E, \Omega, G$ and $p$ be as above, and let $\sigma$ be a permutation of the outcome-set $E$. Then we shall say that $g \in G$ implements $\sigma$ iff $p(\alpha g, x)=p(\alpha, \sigma x)$ for all $\alpha \in \Omega$ and all $x \in E$.

Our outcome-separation assumption (3) implies that if $p(\alpha, \sigma x)=p(\alpha, \tau x)$ for all $\alpha \in \Omega$ and all $x \in E$, then $\sigma x=\tau x$, i.e., $\sigma=\tau$. Thus, if $g \in G$ implements a permtutation $\sigma \in \mathfrak{S}(E)$, it implements only one such permutation, which we may denote by $\sigma_{g}$.

Lemma 4.5 Let $\mathbb{H}$ be the set of all group elements $g \in G$ implementing permutations of $E$. Then $\mathbb{H}$ is a subgroup of $G$ and $\sigma: \mathbb{H} \rightarrow \mathfrak{S}(E), g \mapsto \sigma_{g}$, is a homomorphism.

Proof: Let $g, h \in \mathbb{H}$. Then for all $\alpha \in \Omega$ and all $x \in E$, we have

$$
p(\alpha g h, x)=p\left(\alpha g, \sigma_{h} x\right)=p\left(\alpha, \sigma_{g} \sigma_{h} x\right) .
$$

Hence, $g h \in \mathbb{H}$ with $\sigma_{g h}=\sigma_{g} \sigma_{h}$. We also have

$$
p\left(\alpha g^{-1}, x\right)=p\left(\alpha g^{-1}, \sigma_{g} \sigma_{g}^{-1} x\right)=p\left(\alpha g^{-1} g, \sigma_{g}^{-1} x\right)=p\left(\alpha, \sigma^{-1} x\right)
$$

so $g^{-1} \in H$.

Thus, $E$ carries a natural $\mathbb{H}$ action. In what follows, I will simplify the notation by writing $h x$ for $\sigma_{h} x$, where $h \in \mathbb{H}$ and $x \in E$. It is natural to consider cases in which $E$ is transitive as an $\mathbb{H}$-set, meaning that for every $x, y \in E$, there exits at least one $h \in H$ with $h x=y$. This motivates the following

Definition 4.6 (Dynamical classical models) A dynamical classical model (DCM) consists of groups $H \leq G$, a transtive left $H$-set $E$, a convex right $G$-space $\Omega$, and a Markov kernel $p: \Omega \times E \rightarrow[0,1]$ such that $p(\alpha, h x)=p(\alpha h, x)$ for all $\alpha \in \Omega, h \in H$ and $x \in E$. 
Given a DCM as defined above, for each state $\alpha \in \Omega$ define $\widehat{\alpha}: G \rightarrow \Delta(E)$ by

$$
\widehat{\alpha}(g)(x)=p(\alpha g, x) .
$$

We shall say that a DCM is state-determining iff $\alpha \mapsto \widehat{\alpha}$ is injective, i.e., the probability weights $\widehat{\alpha}(g)$ on $\Delta(E)$, as $g$ ranges over $G$, determine the state $\alpha$. Henceforth, I will assume that all DCMs are statedetermining.

Any DCM can be reinterpreted as a symmetric dynamical probabilistic model in a routine way, which we shall now describe. With notation as in Definition 4.6, let $x_{o} \in E$ and set

$$
\mathbb{K}:=\left\{g \in G \mid p\left(\alpha g, x_{o}\right)=p\left(\alpha, x_{o}\right) \forall \alpha \in \Omega\right\} .
$$

Arguing in much the same way as above, it is easy to see that $\mathbb{K}$, too, is a subgroup of $G$. The following slightly extends a result from [26]; see also [27]:

Theorem 4.7 Let $E$ be a set acted upon transitively by a group $H$, let $G$ be any group with $H \leq G$, and let $K$ be any subgroup of $G$ with $K \leq \mathbb{K}$ and $K \cap H=H_{x_{o}}$. Then there is a well-defined $H$-equivariant injection $\phi: E \rightarrow X$ given by $\phi\left(h x_{o}\right)=h K$ for all $h \in H$. Identifying $E$ with $\phi(E) \subseteq X$,

(a) $\mathcal{M}:=\{g \phi(E) \mid g \in G\}$ is a fully G-symmetric test space, and

(b) For every $\alpha \in \Omega$,

$$
\underset{\sim}{\alpha}\left(x_{g}\right):=\alpha\left(g, x_{o}\right)
$$

is a well-defined probability weight on $\mathcal{M}$; and

(c) The mapping $\alpha \mapsto \underset{\sim}{\alpha}$ is a G-equivariant affine injection.

The proof is given in Appendix C.

Thus, the essentially classical picture presented above - a single test (or observable) E, interacting probabilistically with a system with a state-space $\Omega$ and symmetry group $G$ by means of the function $p: \Omega \times E \rightarrow[0,1]$ — can be reinterpreted as a "non-classical" probabilistic model in which $E$ appears as one of many possible tests, provided that a sufficiently large set of permutations of $E$ can be implemented physically by the group $G$. What is more important for our purposes, however, is the (even more) trivial converse: Any symmetric model $A$, and in particular, any (finite-dimensional) quantum model, arises from this construction: simply take $H$ to be the stabilizer in $G(A)$ of a chosen test $E \in \mathcal{M}(A)$ and $K$ to be the stabilizer in $G(A)$ of some chosen outcome $x_{o} \in E$, and we recover $(\mathcal{M}(A), \Omega(A))$ from Theorem 4.7.

To summarize: in the representation above, we have reinterpreted the states as, in effect, random probability measures (or rather, weights) on a fixed test $E$, indexed by the dynamical group $G(A)$. Another way to view this is that each state determines a family of trajectories in the simplex $\Delta(E)$ of classical probability weights on $E$. Given a state $\alpha \in \Omega$ plus the system's actual dynamics, as specified by a choice of one-parameter group $g: \mathbb{R} \rightarrow G(A)$, we obtain a path $\widehat{\alpha}_{t}:=\widehat{\alpha}\left(g_{t}\right)$ in $\Delta(E)$. In this representation, notice,

(a) States are not viewed as probability weights on a non-classical test space, but rather, specify how classical probabilities change over time, given the dynamics.

(b) In general, the trajectories in $\Delta(E)$ arising from states and one-parameter subgroups of $G(A)$ are not governed by flows, that is, there is no one-parameter group of affine mappings $T^{s}: \Delta(E) \rightarrow \Delta(E)$ such that $\widehat{\alpha}_{t+s}=T^{s}\left(\alpha_{t}\right)$. In particular, the observed evolution of probabilities on $E$ is not Markov. In this respect, it is the dynamical, rather than the probabilistic, structure of the DCM that can be regarded as non-classical.

It is also important to note that the representation of a symmetric probabilistic model as a DCM is not in any usual sense a "hidden variables" representation: it invokes no additional structure, but is simply a 
different, and mathematically equivalent, way of viewing the model (in particular, its states) — one that, I am urging, we might nevertheless want to to count as probabilistically "classical": to whatever extent it departs from classicality, it does so with respect to its dynamical, and not with respect to its probabilistic, structure.

\section{Composite models, entanglement and locality}

Thus far, we have been discussing probabilistic models largely in isolation, and largely in abstraction from physics. Once we start to consider probabilistic models in relation to one another, and as representing actual physical systems localized in spacetime, e.g., particles (or laboratories), we encounter a host of new questions regarding compound systems in which two or more component systems occupy causally separate regions of spacetime. In particular, we unavoidably encounter the concepts of entanglement and locality.

In this section, I briefly review how these notions unfold in the context of general probabilistic theories (following [4, 12]), and how the various classical representations discussed above interact with such composites. As we'll see, entangled states arise naturally in this context. There is a sense in which the representations in terms of classical extensions and in terms of semiclassical covers are both obviously non-local, simply because in each case the "classical" state spaces associated with a composite system $A B$ is typically much larger than the Cartesian product of those associated with $A$ and $B$ separately. Thus, for instance, if $S(A)$ stands for the set of dispersion-free probability weights on $\widetilde{\mathcal{M}}(A)$, then $S(A B)$ is generally much larger than $S(A) \times S(B)$. Similarly, unless $\Omega(A)$ or $\Omega(B)$ is a simplex, $\Omega(A B)$ will generally allow for entangled pure states, which essentially just means that $\Omega(A B)_{\text {ext }}$ will be larger than $\Omega(A)_{\text {ext }} \times \Omega(B)$ ext. A more technical notion of "non-locality" in terms of hidden variables is discussed below in subsection 5.2, while the more delicate question of whether composites of DCMs should be regarded as local or not is discussed in section 5.4 .

\subsection{Composites of probabilistic models}

Suppose $A$ and $B$ are probabilistic models. A joint probability weight on the test spaces $\mathcal{M}(A)$ and $\mathcal{M}(B)$ is a function $\omega: X(A) \times X(B) \rightarrow[0,1]$ that sums to 1 on each product test $E \times F$, where $E \in \mathcal{M}(A)$ and $F \in \mathcal{M}(B)$. Joint probability weights can be understood as probability weights on the test space

$$
\mathcal{M}(A) \times \mathcal{M}(B):=\{E \times F \mid E \in \mathcal{M}(A), F \in \mathcal{M}(B)\}
$$

consisting of all product tests. If $\alpha$ and $\beta$ are probability weights on $A$ and $B$, we can form a joint probability weight $\alpha \otimes \beta$ on $A$ and $B$, by the obvious recipe $(\alpha \otimes \beta)(x, y)=\alpha(x) \beta(y)$ for outcomes $x \in X(A)$ and $y \in X(B)$.

Definition 5.1 (Non-signaling joint probability weights) A joint probability weight $\omega$ on $\mathcal{M}(A)$ and $\mathcal{M}(B)$ is non-signaling iff its marginal (or reduced) probability weights, given by

$$
\omega_{2}(y):=\omega(E \times\{y\}) \text { and } \omega_{1}(x):=\omega(\{x\} \times F)
$$

are well-defined, i.e., independent of the choice of $E \in \mathcal{M}(A)$ and $F \in \mathcal{M}(B)$, respectively.

To the extent to which we think of the tests $E \in \mathcal{M}(A)$ as representing the physical actions performable on the system represented by $A$ (in the sense that to perform a test is to do something to the system, and then observe a result), and similarly for $B$, the non-signaling condition on $\omega$ tells us that no action performable on $A$ can have any statistically detectable influence on $B$, and vice versa. Thus, the no-signaling condition is the locality condition appropriate to states qua probability weights. 
A non-signaling joint probability weight, having well-defined marginals, also has well-defined conditional probability weights given by

$$
\omega_{2 \mid x}(y):=\frac{\omega(x, y)}{\omega_{2}(y)} \text { and } \omega_{1 \mid y}(x):=\frac{\omega(x, y)}{\omega_{1}(x)}
$$

(with, say, the convention that both are zero if their denominators are). The marginal states can be recovered as convex combinations of these conditional states, in a version of the law of total probability:

$$
\omega_{1}=\sum_{y \in F} \omega_{2}(y) \omega_{1 \mid y} \text { and } \omega_{2}=\sum_{x \in E} \omega_{1}(x) \omega_{2 \mid x} .
$$

We would certainly want these conditional and (hence) marginal states to belong to the designated statespaces of each component of a reasonable composite model.

Definition 5.2 Given probabilistic models $A$ and $B$, let $A \otimes B$ denote the probabilistic model with test space $\mathcal{M}(A \otimes B):=\mathcal{M}(A) \times \mathcal{M}(B)$ and state-space $\Omega(A \otimes B)$ consisting of all non-signaling joint states $\omega$ on $\mathcal{M}(A) \times \mathcal{M}(B)$ having conditional (and hence, marginal) states belonging to $\Omega(A)$ and $\Omega(B)$.

This is essentially the maximal (or injective) tensor product of $\Omega(A)$ and $\Omega(B)[4,20]$. Since it does not allow for non-product outcomes, it is too small to be generally useful as a model of composite systems, but it does afford an easy way to define something more general:

Definition 5.3 (Non-signaling composites [4]) A non-signaling composite of probabilistic models $A$ and $B$ is a model $A B$, together with

(a) an outcome-preserving interpretation $\phi: A \otimes B \rightarrow A B$, taking each pair of outcomes $x \in$ $X(A)$ and $y \in X(B)$ to a product outcome $x y:=\phi(x, y)^{8}$, and

(b) a bi-affine mapping $\Omega(A) \times \Omega(B) \rightarrow \Omega(A B)$ taking states $\alpha \in \Omega(A)$ and $\beta \in \Omega(B)$ to a product state $\alpha \otimes \beta$ such that $(\alpha \otimes \beta)(x y)=\alpha(x) \beta(y)$ for all $x \in X(A), y \in X(B)$.

Henceforth, by a composite of two probabilistic models, I will always mean a non-signaling composite in this sense.

Example 5.4 Consider quantum probabilistic models $A(\mathcal{H})$ and $A(\mathcal{K})$. If we take $A B$ to be $A(\mathcal{H} \otimes \mathcal{K})$, then we have a natural map $\phi: X(\mathcal{H}) \times X(\mathcal{K}) \rightarrow X(\mathcal{H} \otimes \mathcal{K})$, given by $\phi(x, y)=x \otimes y$. It is easy to check that this is an interpretation from $A(\mathcal{H}) \times A(\mathcal{K})$. We also have an affine mapping $\Omega(\mathcal{H}) \times \Omega(\mathcal{K}) \rightarrow \Omega(\mathcal{H} \otimes \mathcal{K})$ given by $\left(\alpha_{T}, \alpha_{W}\right) \mapsto \alpha_{T \otimes W}$ (where, recall, $\alpha_{T}$ represents the probability weight determined by the density operator $T$ ). It is easy to see that these mappings satisfy the conditions above.

If $A B$ is a composite of models $A$ and $B$ as in definition 5.3, every state $\omega \in \Omega(A B)$ pulls back along the designated interpretation $\phi: A \otimes B \rightarrow A B$ to a nonsignaling joint probability weight $\phi^{*}(\omega)(x, y)=\omega(x y)$ on $\mathcal{M}(A) \times \mathcal{M}(B)$ with conditional and marginal weights belonging to $\Omega(A)$ and $\Omega(B)$. In general, this joint probability weight does not determine the state $\omega$.

Definition 5.5 (Local tomography) $A$ composite $A B$ of probabilistic models $A$ and $B$, with designated interpretation $\phi: A \otimes B \rightarrow A B$, is locally tomographic iff the mapping $\phi^{*}: \Omega(A B) \rightarrow \Omega(A \times B)$ is injective.

\footnotetext{
${ }^{8} \mathrm{~A}$ more general definition would drop the requirement that $\phi$ be outcome-preserving, thus allowing for the possibility that for some outcomes $x$ and $y, \phi(x, y)=: x y$ might be a non-trivial (and even possibly empty) event of $A B$. We will not need this generality here.
} 
It is easy to show that, for finite-dimensional probabilistic models $A$ and $B, A B$ is locally tomographic iff the mapping $\otimes: \Omega(A) \times \Omega(B) \rightarrow \Omega(A B)$ extends to a linear isomorphism $\mathbf{V}(A) \otimes \mathbf{V}(B) \simeq \mathbf{V}(A B)$. Mathematically, then, local tomography is a great convenience. However, given its failure for real and quaternionic QM, unless we are looking for an excuse to rule out these variants of QM (and see, e.g., [3] for reasons why we might not want to do so), it is probably best to avoid taking local tomography as an axiom.

It is natural to ask that a probabilistic theory be closed under some rule of composition, in the sense of Definition 5.3, that is consonant with the theory's categorical structure. A way to make this precise is to ask that a probabilistic theory be a symmetric monoidal category. See [4] for further discussion.

\subsection{Entanglement}

If $A B$ is a composite of models $A$ and $B$, then any convex combination of product states, say $\sum_{i} t_{i} \alpha_{i} \otimes \beta_{i}$ or, more generally,

$$
\int_{\Lambda}\left(\alpha_{\lambda} \otimes \beta_{\lambda}\right) d \mu(\lambda)^{9}
$$

is said to be separable. Such states can (in principle) be prepared by randomly selecting product states $\alpha_{\lambda}$ and $\beta_{\lambda}$ for $A$ and $B$, respectively, in a classically correlated way. States not of this form are said to be entangled. The existence, and the basic properties, of entangled states are rather generic features of probabilistic models having non-simplex state spaces. In particular, we have the following

Lemma 5.6 Let $\omega$ be any non-signaling state on $A B$. Then

(a) If $\alpha \otimes \beta$ is pure, then so are $\alpha$ and $\beta$;

(b) If either of the marginal states $\omega_{1} \in \Omega(A)$ or $\omega_{2} \in \Omega(B)$ is pure, then $\omega=\omega_{1} \otimes \omega_{2}$;

(c) Hence, if $\omega$ is entangled, then $\omega_{1}$ and $\omega_{2}$ are mixed.

Proof: See [4] (and note that the arguments are virtually identical to the familiar ones in the context of $\mathrm{QM}) \cdot{ }^{10}$

When $A$ and $B$ are semiclassical, the test space $\mathcal{M}(A \otimes B)$ of Defintion 5.2 is again semiclassical. In this situation, we have lots of dispersion-free joint states. However:

Lemma 5.7 Let $A$ and $B$ be models with semiclassical test spaces $\mathcal{M}(A)$ and $\mathcal{M}(B)$, and let $\omega \in$ $\operatorname{Pr}(\mathcal{M}(A \times B))$. If $\omega$ is non-signaling and dispersion-free, then $\omega=\delta \otimes \gamma$ where $\delta$ and $\gamma$ are dispersion-free.

Proof: Suppose $\omega$ is dispersion-free. Since $\omega$ is also non-signaling, it has well-defined marginal states, which must obviously also be dispersion-free, hence, pure. But by Lemma 5.6, a non-signaling state with pure marginals is the product of these marginals.

It follows that any average of non-signaling, dispersion-free states is separable.

\footnotetext{
${ }^{9}$ where $\Lambda$ is a measurable space and $\mu$ is a probability measure thereon, and where the integral exists in the obvious sense that

$$
\left.\left(\int_{\Lambda} \alpha_{\lambda} \otimes \beta_{\lambda}\right) d \mu(\lambda)\right)(z)=\int_{\Lambda}\left(\alpha_{\lambda} \otimes \beta_{\lambda}\right)(z) d \mu(\lambda)
$$

${ }^{10}$ As an historical note, both of these points were first noted in this generality, but without any reference to entanglement, in a pioneering paper of Namioka and Phelps [20] on tensor products of compact convex sets. They were rediscovered, and connected with entanglement, by Kläy [17].
} 


\subsection{Locality and hidden variables}

In the literature, a (contextual) local hidden variable model for a bipartite probability weight $\omega$ is usually taken to consist of a measurable space $\Lambda$, a probability measure $\mu$ thereon, and a pair of response functions $p_{A}$ and $p_{B}$ such that, such that for all tests $E \in \mathcal{M}(A), F \in \mathcal{M}(B)$ and all outcomes $x \in E$ and $y \in F \in \mathcal{M}(B), p(x \mid E, \lambda)$ is the probability of $x$ given the parameter $\lambda$ and the choice of measurement $E$, and similarly for $p(y \mid F, \lambda)$. These are required to satisfy

$$
\omega(x, y)=\int_{\Lambda} p_{A}(x \mid E, \lambda) p(y \mid F, \lambda) d \mu(\lambda)
$$

so that we can interpret the joint probability $\omega(x, y)$ as resulting from a classical correlation (given by $\mu$ ) together with the local response functions $p_{A}$ and $p_{B}$.

As discussed in section 3.3, it is straightforward to reinterpret the functions $p_{A}$ and $p_{B}$ in terms of probability weights on $\widetilde{\mathcal{M}}(A)$ and $\widetilde{\mathcal{M}}(B)$, respectively, by writing $p_{A}(x \mid E, \lambda)$ as $p_{A, \lambda}(x, E)$ and $p_{B}(y \mid F, \lambda)$ as $p_{B, \lambda}(y, F)$ (emphasizing that $\lambda$ can be treated as merely an index). We then have

$$
\omega(x, y)=\left(\int_{\Lambda} p_{A, \lambda} \otimes p_{B, \lambda} d \mu(\lambda)\right)((x, E),(y, F)),
$$

independently of $E$ and $F$. In other words, $\omega$ has a local HV model if, and only if, it is separable, in the integral sense, when regarded as a joint probability weight on $\widetilde{\mathcal{M}}(A \otimes B)$, where $A \otimes B$ is the composite of Definition 5.2.

Remark: A first reaction to this observation might be that it must be wrong, as it's well-known that there exist entangled Werner states that nevertheless have local HV representations [25]. The subtlety here (such as it is) resides in the fact that whether a state is separable or entangled depends on what "local" state-spaces are in play. Here, we are expressing $\omega$ as a weighted average of products of "contextual" states, whereas in standard discussions of quantum entanglement, a state is separable iff it can be expressed as a weighted average of products of quantum (in particular, non-contextual) states of the component systems.

\subsection{Composites of dynamical models}

Since dynamical classical models are simply another way of looking at symmetric probabilistic models, in principle they can be composed in the same way as the latter. I have discussed this in some technical detail elsewhere [27]. Without going into such detail, I want to make a few remarks on the sense in which composites of DCMs can be regarded as local. Suppose, then, that

$$
A=\left(\Omega(A), G(A), H(A), E(A), p_{A}\right) \text { and } B=\left(\Omega(B), G(B), H(B), E(B), p_{B}\right)
$$

are two DCMs as defined in Section 4.2, and suppose that $A B$ is a DCM serving as a composite of these. This will require, at a minimum, that $E(A B)=E(A) \times E(B)$, and that we have functions

$$
\otimes: \Omega(A) \times \Omega(B) \rightarrow \Omega(A B) \text { and } \otimes: G(A) \times G(B) \rightarrow G(A B)
$$

with the former bi-affine and the latter a group homomorphism, such that $(G(A) \otimes G(B)) \cap H(A B) \leq$ $H(A) \otimes H(B)$ and

$$
(\alpha \otimes \beta)(g \otimes h)=\alpha g \otimes \beta h
$$

for all $(\alpha, \beta) \in \Omega(A) \times \Omega(B)$ and all $(g, h) \in G(A) \times G(B)$. Additional conditions are necessary to ensure that a non-signaling condition is satisfied, but this is enough for present purposes.

Examples 5.8 (a) By the minimal classical dynamical model associated with a finite set $E$, I mean the $\operatorname{DCM} A(E)$ with $\Omega(A(E))=\Delta(E), G(A(E))=H(A(E))=\mathfrak{S}(E)$ (the symmetric group of all bijections 
$E \rightarrow E)$ and $p_{A(E)}(\alpha, x)=\alpha(x)$. Now given two finite sets, then the minimal DCM $A(E \times F)$ is a composite of $A(E)$ and $A(F)$ with the maps $\otimes: \Delta(E) \times \Delta(F) \rightarrow \Delta(E \times F)$ and $\otimes \mathfrak{S}(E) \times \mathfrak{S}(F) \rightarrow \mathfrak{S}(E \times F)$ the obvious ones, that is,

$$
(\alpha \otimes \beta)(x, y)=\alpha(x) \beta(y) \text { and }(g, h)(x, y)=(g x, h y)
$$

for all $(x, y) \in E \times F,(\alpha, \beta) \in \Delta(E) \times \Delta(F)$ and $(g, h) \in \mathfrak{S}(E) \times \mathfrak{S}(F)$.

(b) Let $A$ and $B$ be quantum-mechanical systems associated with Hilbert spaces $\mathcal{H}_{A}$ and $\mathcal{H}_{B}$. Fixing orthonormal bases $E \in \mathcal{M}\left(\mathcal{H}_{A}\right)$ and $F \in \mathcal{M}\left(\mathcal{H}_{B}\right)$, we can regard $A$ and $B$ as DCMs with $\Omega(A)$ and $\Omega(B)$ the spaces of density operators on $\mathcal{H}_{A}$ and $\mathcal{H}_{B}$, respectively, $G(A)=\mathcal{U}\left(\mathcal{H}_{A}\right)$ and $G(B)=\mathcal{U}\left(\mathcal{H}_{B}\right)$, and with

$$
p_{A}(W, x)=\operatorname{Tr}\left(W p_{x}\right)=\langle W x, x\rangle
$$

and similarly for $p_{B}$. Identifying $E \times F$ with the product basis $E \otimes F$ in $\mathcal{M}\left(\mathcal{H}_{A} \otimes \mathcal{H}_{B}\right)$, we can regard the quantum system associated with $\mathcal{H}_{A} \otimes \mathcal{H}_{B}$ as a DCM in the same way. In this case the maps $\otimes$ referred to above are the obvious ones: given unitaries $U \in \mathcal{U}\left(\mathcal{H}_{A}\right)$ and $V \in \mathcal{U}\left(\mathcal{H}_{B}\right)$, we have a untary $U \otimes V$ on $\mathcal{H}_{A} \otimes \mathcal{H}_{B}$, and if $W_{A}$ and $W_{B}$ are density operators on $\mathcal{H}_{A}$ and $\mathcal{H}_{B}$ respectively, the $W_{A} \otimes \mathcal{W}_{B}$ is the corresponding density operator on $\mathcal{H}_{A} \otimes \mathcal{H}_{B}$.

As discussed above, the prevailing technical definition of a local probabilistic theory is one in which composite systems admit local hidden-variable models (ideally, in a functorial way, though this point is rarely discussed). More generally, one would like to say that a probabilistic theory is local iff composite systems admit "local" classical representations, in some well-defined (but reasonably general) sense. As I've mentioned, the classical extensions and the classical representations associated with semiclassical covers, discussed in Sections 3.2 and 3.3, are certainly not local in any reasonable sense. What about composites of DCMs? In what sense are, or aren't, these to be regarded as local classical representations of symmetric probabilistic models? As Example 5.4 and the second example above illustrate, the state space $\Omega(A B)$ will in general be significantly larger than the convex hull of $\Omega(A) \times \Omega(B)$, in the sense that there will be entangled states. In this weak sense, such a composite will generally be "non-local". On the other hand, it is not entirely clear how to discuss the locality of a composite of dynamical classical models as a classical representation, since it involves only a single, classical measurement $E(A) \times E(B)$, and invokes no hidden variables, non-local or otherwise - unless, of course, we wish to regard the symmetries in $G(A B)$ as "hidden variables". In that case, the "non-locality" presumably rests in the fact that the group $G(A B)$ will typically be a great deal larger than $G(A) \times G(B)$. This is true, of course, for quantum models, where the unitary group $\mathcal{U}\left(\mathcal{H}_{A} \otimes \mathcal{H}_{B}\right)$ is larger than $\mathcal{U}\left(\mathcal{H}_{A}\right) \otimes \mathcal{U}\left(\mathcal{H}_{B}\right)$. But it is also true for minimal classical dynamical models, since for sets $E$ and $F$, the symmetric group $\mathfrak{S}(E \times F)$ is larger than $\mathfrak{S}(E) \times \mathfrak{S}(F)$.

\section{Conclusion}

Many of the observations collected above are well known, at least folklorically. My aim in bringing them together, in the particular way that I have, has been to draw attention to some points that I believe follow from these observations collectively, and that I believe are somewhat less widely appreciated than they might be:

(a) Classicality in the strict (that is, unrestricted) Kolmogorovian sense, is manifestly a contingent matter, since it is not a point of logical necessity that all experiments should be compatible. To the extent that probability theory is meant to be a completely general and a priori study of reasoning in the face of uncertainty or chance, it isn't Kolmogorovian, nor is it quantum-mechanical: it is, rather, the study of what I've here called probabilistic theories generally (whether formulated as I have done here, or in some roughly similar way).

(b) Within such a framework, what we ought to mean by saying that a probabilistic model or probabilistic theory is "classical" is not entirely obvious. For some values of "classical", every probabilistic theory 
is, or can be represented as, a classical one, at the cost of contextuality and, where we are dealing with a non-signaling theory, also of locality. Moreover, for certain kinds of theories (fully symmetric ones), there is a broad sense of "classical" in which such a theory simply is classical, without any need to invoke hidden variables, contextual, non-local or otherwise. Or at least, this is true for the theory's probabilistic structure: any departure from "classicality" is dynamical. Finite-dimensional quantum mechanics is such a theory.

(c) The selection of a class of probabilistic models - a probabilistic theory - must rest on some contingent assumptions about the nature of the entities one seeks to model. Quantum mechanics, as a probabilistic theory, is contingent in this way; so is (unrestricted, Kolmogorovian) classical probability theory, and the two are not the same. In this limited and unremarkable sense, quantum mechanics is (of course) a non-classical probabilistic theory.

I don't know how much, if any, of this Pitowsky would have endorsed. Regarding (b), at least, he was very clear:

We can always avoid the radical view of probability by adopting a non-local, contextual hidden variables theory such as Bohm's. But then I believe, the philosophical point is missed. It is like taking Steven Weinberg's position on space-time in general relativity: There is no non-flat Riemannian geometry, only a gravitational field defined on a flat space-time that appears as if it gives rise to geometry ... I think that Weinberg's point and also Bohm's theory are justified only to the extent that they yield new discoveries in physics (as Weinberg certainly hoped). So far they haven't.

I have no issue to take with this, as far as it goes: we are always free to reject a classical representation of a probabilistic theory by objecting to the additional "classical" structure as "non-physical". But I would also want to say that doing so makes the theory a physical theory, and not a theory of probability. Or, to put it differently, whether one wants to take, for example, a set of contextual hidden variables, or a privileged observable, seriously in formulating a given physical theory is a pragmatic question about the best (the most elegant, the most convenient, the most fruitful) way to formulate mathematical models of a particular sort of physical situation. It is also (in particular!) a metaphysical question about what ontology one wants to admit in framing such a model. Such decisions are important; but they are not decisions about what kind of probability theory to use.

Pitowsky also says (emphasis mine):

In this paper, all we have discussed is the Hilbert space formalism. I have argued that it is a new kind of probability theory that is quite devoid of physical content, save perhaps the indeterminacy principle which is built into axiom $\mathrm{H} 4$. Within this formal context there is no explication of what a measurement is, only the identification of "observables" as Hermitian operators. In this respect the Hilbert space formalism is really just a syntax which represents the set of all possible outcomes, of all possible measurements.

Here, of course, I disagree. The "syntax" of quantum probability theory is very definitely not devoid of physical content. Reconstructions of QM from various sets of postulates, ranging from the Piron-AmemiyaAraki-Solèr reconstruction cited by Pitowsky (see [16]), to the various more recent finite-dimensional reconstructions (e.g., $[5,9,10,19,28])$ have helped us understand the physical content of quantum theory more clearly, by isolating operationally meaningful principles that dictate that syntax. These principles invariably include some that are manifestly contingent, at least to the same degree that the classical co-measurability principle is contingent.

The final lines of Pitowsky's paper crystalize what I find problematic in his proposal. (Again, the emphasis is mine.) 
However, there is a structure to the set of events. Not only does each and every type of measurement yield a systematic outcome; but also the set of all possible outcomes of all measurements — including those that have been realized by an actual recording — hang together tightly in the structure of [the projection lattice] $L(\mathcal{H})$. This is the quantum mechanical structure of reality.

Taken in the context of Pitowsky's claim that QM represents a new, non-classical theory of probability, this suggests a certain naturalism regarding probability theory (echoing von Neumann/Birkhoff's naturalism regarding logic): that there is a true probability theory, which is the one that works best, and most broadly, to describe the world we actually live in. If the world really is, at every scale and in every way, described by some version of quantum theory, so that there just are no actual events that can't be described as effects in (say) some grand von Neumann algebra, then quantum probability theory is this true, correct probability theory, of which classical probability theory is simply a limiting or special case (where $\hbar$ tends to zero, or where we restrict attention to a set of commuting observables).

But this view seems to me misleading: not because naturalism per se is wrong (even about mathematics), but because it forgets that probability theory has a methodological as well as a metaphysical importance: it is not simply part of our scientific description of the world, but is part of the framework within which we think about, criticize, and (often) revise that description. In order for it to play this role, it can not be tied to any particular physical theory. This is why I think it is vital to maintain the distinction between probability theory and the various probabilistic theories that it studies. There's no such thing as a probablility theory, classical or otherwise: there is only probability theory. There can be a correct probabilistic theory of the world — and perhaps it's quantum.

\section{References}

[1] E. Alfsen. Convex Sets and Boundary Integrals. Springer, 1971.

[2] G. Bacciagaluppi. Classical extensions, classical representations, and bayesian updating in quantum mechanics. arXiv:quant-ph/0403055v1, 2004.

[3] J. Baez. Division algebras and quantum theory. Foundations of Physics, 42:819-855, 2012. (arXiv:1101.5690).

[4] H. Barnum and A. Wilce. Post-classical probability theory. In G. Chiribella and R. Spekkens, editors, Quantum Theory: Informational Foundations and Foils. Springer, 2016.

[5] H. Barnum, M. Mueller, and C. Ududec. Higher-order interference and single-system postulates characterizing quantum theory. New J. Physics, 16, 2014. (arXiv:1403.4147).

[6] E. Beltrametti and S. Bugajski. A classical extension of quantum mechanics. Journal of Physics A, 28:3329, 1995.

[7] J. Bub and I. Pitowsky. Two dogmas about quantum mechanics. In S. Saunders, J. Barrett, A. Kent, and D. Wallace, editors, Many Worlds? Everett, Quantum Theory and Reality, chapter 14, pages 433-459. Oxford, 2012. (arXiv:0712.4258).

[8] S. Bush, K.-E. Hellwig, and W. Stulpe. On classical representations of finite-dimensional quantum mechanics. International Journal of Theoretical Physics, 32, 1993.

[9] G. Chiribella, M. D'Ariano, and P. Perinotti. Informational derivation of quantum theory. Physical Review A, 84:012311, 2011.

[10] B. Dakič and C. Brukner. Quantum theory and beyond: is entanglement special? In H. Halvorson, editor, Deep Beauty: Understanding the Quantum World through Mathematical Innovation. Cambridge University Press, 2011. (arXiv:0911.0695). 
[11] D. J. Foulis and C. H. Randall. Manuals, morphisms and quantum mechanics. In A. R. Marlowe, editor, Mathematical Foundations of Quantum Theory. Academic Press, 1978.

[12] D. J. Foulis and C. H. Randall. Empirical logic and tensor products. In H. Neumann, editor, Interpretations and Foundations of Quantum Mechanics. B.I.-Wissenshaftsverlag, 1981.

[13] L. Hardy. Quantum theory from five reasonable axioms. arXiv:quant-ph/0101012, 2001.

[14] Hellwig and Singer. "classical" in terms of general statistical models. In V. Dodonov and V. Man'ko, editors, Group Theoretical Methods in Physics, volume 382 of Lecture Notes in Physics. Springer, 1991.

[15] A. Holevo. Probabilistic and statistical aspects of quantum theory. North Holland, 1982.

[16] S. Holland. Orthomodularity in infinite dimensions: a theorem of m. solèr. Bulletin of the American Mathematical Society, 32:205-234, 1995. (arXiv:math/9504224).

[17] M. Kläy. Einstein-podolsky-rosen experiments: the structure of the probability space i, ii. Foundations of Physics Letters, 1:205-244, 1988.

[18] B. Lindsay. Mixture Models: Theory, Geometry and Applications, volume 5 of NSF-CBMS Regional Conference Series in Probability and Statistics. Institute of Mathematical Statistics/American Statistical Association, 1995.

[19] Ll. Masanes and M. Müller. A derivation of quantum theory from physical requirements. New Journal of Physics, 13, 2011. (arXiv:1004.1483).

[20] I. Namioka and R. Phelps. Tensor products of compact convex sets. Pacific J. Math, 3:469-480, 1969.

[21] I. Pitowsky. Quantum mechanics as a theory of probability. In W. Demopolous, editor, Physical Theory and its Interpretation: Essays in Honor of Jeffrey Bub, Western Ontario Series in Philosophy of Science. Kluwer, 2005. (arXiv: 0510095).

[22] S. Popescu and D. Rohrlich. Quantum nonlocality as an axiom. Foundations of Physics, 24:379-285, 1994.

[23] R. Spekkens. Contextuality for preparations, transformations and unsharp measurements. Phys. Rev. $A, 71,2005$. (arXiv:quant-ph/0406166).

[24] V. S. Varadarajan. The Geometry of Quantum Theory. Springer, 2 edition, 1985.

[25] R. Werner. Quantum states with einstein-podolsky-rosen correlations admitting a hidden-variable model. Phys. Rev. A, 40:4277, 1989.

[26] A. Wilce. Symmetry and topology in quantum logic. International Journal of Theoretical Physics, 44: 2303-2316, 2005.

[27] A. Wilce. Symmetry and composition in generalized probabilistic theories. arXiv:0910.1527, 2009.

[28] A. Wilce. Conjugates, filters and quantum mechanics. arXiv:1206.2897, 2012.

[29] A. Wilce. Quantum logic and probability theory. Stanford Encyclopedia of Philosophy, 2017. (https://plato.stanford.edu/archives/spr2017/entries/qt-quantlog/). 


\section{A Common refinements}

Let $A$ be a probabilistic model, and let $E, F \in \mathcal{M}(A)$. Recall that $E$ refines $F$, or that $F$ is a coarsening of $E$, if there exists a surjection $f: E \rightarrow F$ such that, for every $y \in F$ and every state $\alpha \in \Omega$,

$$
\alpha(y)=\alpha\left(f^{-1}(y)\right) .
$$

When this is so, we write $E \preceq F$, and say that $f$ is a coarsening map.

Definition A.1 A set $\Omega$ of probability weights on a test space $\mathcal{M}$ separates compatible events iff, for every test $E \in \mathcal{M}$ and any pair of distinct events $a, b \subseteq E$, there exists a weight $\alpha \in \Omega$ with $\alpha(a) \neq \alpha(b)$.

Note that if $A$ is unital, $\Omega(A)$ separates compatible events. Indeed, it is sufficient that, for every $x \in E$, there exist a state $\alpha \in \Omega(A)$ with $\alpha(x)>1 / 2$.

Lemma A.2 Let $\Omega(A)$ separate compatible events. Then

(a) There exists at most one coarsening map $f: E \rightarrow F$;

(b) If there exist coarsening maps $f: E \rightarrow F$ and $g: F \rightarrow E$, then $f$ and $g$ are bijections and $g=f^{-1}$;

(c) If $f: G \rightarrow E$ and $g: G \rightarrow F$ are coarsening maps and $x \in E \cap F$, then $f^{-1}(x)=g^{-1}(x) \subseteq G$.

Proof: (a) If $f, g: E \rightarrow F$ are coarsening maps, where $E \preceq F$ in $\mathcal{M}(A)$, then for every $y \in F$ we have $\alpha\left(f^{-1}(y)\right)=\alpha(y)=\alpha\left(g^{-1}(y)\right)$ for every $\alpha \in \Omega(A)$. Hence, $f^{-1}(y)=g^{-1}(y)$ for every $y \in F$, whence, $f=g$. Now (b) follows, since the composition of coarsening maps is a coarsening map. For (c), observe that if $x \in E \cap F$, then for every state $\alpha$ we have $\alpha\left(f^{-1}(x)\right)=\alpha(x)=\alpha\left(g^{-1}(x)\right)$. Since $\Omega$ separates compatible events, $f^{-1}(x)=g^{-1}(x)$.

From now on, assume $\Omega(A)$ separates compatible events. We will write $f_{E, F}$ for the unique coarsening map $f: E \rightarrow F$, if one exists.

Suppose now that $E, F \in \mathcal{M}(A)$ have a common refinement, that is, that there exists a test $G \in \mathcal{M}(A)$ with $G \preceq E$ and $G \preceq F$. Then we have a natural surjection $\phi: G \rightarrow E \times F$, namely

$$
\phi(z)=\left(f_{G, F}(z), f_{G, E}(z)\right) .
$$

If $\alpha \in \Omega$, then we have a probability weight on $E \times F$ given by

$$
\phi_{*}(\alpha):=\alpha \circ \phi^{-1} .
$$

This assigns to $(x, y) \in E \times F$ the probability

$$
\alpha\left(\phi^{-1}(x, y)\right)=\alpha\left(a_{x} \cap b_{y}\right), \text { where } a_{x}=f_{G, E}^{-1}(x) \text { and } b_{y}=f_{G, F}^{-1}(y) .
$$

It is easy to check that $\sum_{y \in F} \phi_{*}(\alpha)(x, y)=\alpha(x)$ and $\sum_{x \in E} \phi_{*}(\alpha)(x, y)=\alpha(y)$, i.e., $\phi_{*}(\alpha)$ has the "right" marginals to explain the probabilities that $\alpha$ assigns to $E$ and $F$. In this sense, $G$ can be regarded as a joint measurement of $E$ and $F$.

Definition A.3 $A$ is a refinement ideal iff every pair of tests in $\mathcal{M}(A)$ has a common refinement.

In other words, $A$ is a refinement ideal iff the preordered set $(\mathcal{M}, \preceq)$ is downwardly directed. Define

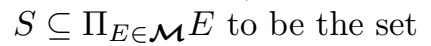

$$
S=\left\{\boldsymbol{x}=\left(x_{E}\right) \in \Pi_{E \in \mathcal{M}(A)} \mid \forall E \preceq F f_{E, F}\left(x_{E}\right)=x_{F}\right\},
$$


i.e., the inverse limit of $\mathcal{M}$, regarded as a small category under coarsening maps. As long as $\mathcal{M}$ is locally finite, one can show that this is non-empty (a consequence of the compactness of $\Pi_{E \in \mathcal{M}} E$ ). For each test $E \in \mathcal{M}(A)$, define $f_{E}: S \rightarrow E$ by $f_{E}(x)=x_{E}$, and for any $x_{o} \in X(A)$, let

$$
\left[x_{o}\right]:=\left\{\boldsymbol{x} \in S \mid \forall E \in \mathcal{M}(A) x_{o} \in E \Rightarrow x_{E}=x_{o}\right\} .
$$

Note that, by Lemma A.2, if $x \in E \cap F$, then for every $\boldsymbol{x} \in S, x_{E}=x_{o}$ iff $x_{F}=x_{o}$; hence, $\left[x_{o}\right] \neq \emptyset$. If $a \in \mathcal{E} \boldsymbol{v}(A)$, let $\llbracket a \rrbracket:=\{[x] \mid x \in a\}$. Notice that $\bigcup \llbracket a \rrbracket=f_{E}^{-1}(a)$ where $E$ is any test with $a \subseteq E$.

Recall that, if $E$ and $F$ are partitions of a set $S, E$ is said to refine $F$ iff for every $y \in F$, there is some $a \subseteq E$ with $y=\cup a$. I will write $E \sqsubseteq F$ to indicate this. If $\mathcal{M}$ is a collection of partitions of a set $S$ such that every pair of partitions in $\mathcal{M}$ has a common refinement in this sense, then I will say that $\mathcal{M}$ is a refinement ideal of partitions.

Lemma A.4 If $A$ is a locally finite refinement ideal, then with $S$ the projective limit of $\mathcal{M}(A)$ in (5), for every test $E \in \mathcal{M}(A), \llbracket E \rrbracket$ is a partition of $S$, and if $E \preceq F$ then $\llbracket E \rrbracket \sqsubseteq \llbracket F \rrbracket$. Hence, $\llbracket \mathcal{M}(A) \rrbracket:=\{\llbracket E \rrbracket \mid E \in$ $\mathcal{M}(A)\}$ is a refinement ideal of partitions. Moreover, the mapping $\phi: x \mapsto[x]$ defines an isomorphism of test spaces from $\mathcal{M}(A)$ to $\llbracket \mathcal{M}(A) \rrbracket$.

Proof: That $\llbracket E \rrbracket$ is a partition of $S$ is clear from the definitions. If $E \preceq F$, let $y \in F$, and set $a=f_{E, F}^{-1}(y) \subseteq$ $E$. Thus, $\llbracket a \rrbracket \subseteq \llbracket E \rrbracket$. Now

$$
\bigcup \llbracket a \rrbracket=f_{E}^{-1}(a)=f_{E}^{-1} \circ f_{E, F}^{-1}(x)=\left(f_{E, F} \circ f_{E}\right)^{-1}(x)=f_{F}^{-1}(y)=[y] .
$$

Thus, every cell in the partition $\llbracket F \rrbracket$ is a union of cells of $\llbracket E \rrbracket$, i.e., $E \sqsubseteq F$. Since every pair of tests in $\mathcal{M}(A)$ have a common refinement with respect to $\Omega(A)$, it follows that $\llbracket \mathcal{M}(A) \rrbracket$ is a refinement ideal of partitions. It is clear that $\phi: x \mapsto[x]$ is an outcome-preserving, positive interpretation from $\mathcal{M}(A)$ onto $\llbracket \mathcal{M}(A) \rrbracket$. It remains to show it's injective. Let $x, y \in X(A)$ with $[x]=[y]$. Supposing that $x \in E \in \mathcal{M}(A)$ and $y \in F \in \mathcal{M}(A)$, let $G \preceq E, F$. Then $f_{G, E}^{-1}(x)=f_{G, F}^{-1}(y)$, so for all $\alpha \in \Omega(A)$, we have

$$
\alpha(x)=\alpha\left(f_{G, E}^{-1}(x)\right)=\alpha\left(f_{G, F}^{-1}(y)\right)=\alpha(y) .
$$

By our standing assumption that states in $\Omega(A)$ separate outcomes in $X(A), x=y$.

Now let $\mathcal{M}$ be a test space of partitions of a set $S$, and let $\mathcal{M}$ be a refinement ideal with respect to ordinary refinement of partitions. Define $\phi: \mathcal{E} \boldsymbol{v}(\mathcal{M}) \rightarrow \mathcal{P}(S)$ by $\widehat{a}=\cup a$ for all $a \in \mathcal{E} \boldsymbol{v}(\mathcal{M})$. Note here that an event $a$ is a subset of a finite partition of $S$, that is, a finite, pairwise disjoint set of non-empty subsets of $S$. Now let

$$
\Sigma:=\{\widehat{a} \mid a \in \mathcal{E} \boldsymbol{v}(\mathcal{M})\} .
$$

Lemma A.5 $\Sigma$ is an algebra of sets on $S$.

Proof: It is easy to see that if $a, b \subseteq E \in \mathcal{M}, \widehat{a} \cap \widehat{b}=\widehat{a \cap b}, \widehat{a \cup b}=\widehat{a} \cup \widehat{b}$, and $\widehat{a}^{c}=\widehat{E \backslash a}$. Thus, the set $\Sigma_{E}:=\{\widehat{a} \mid a \subseteq E\}$ is a subalgebra of $\mathcal{P}(S)$. If $G \sqsubseteq E$, then the definition of refinement tells us that $\Sigma_{E} \subseteq \Sigma_{G}$. Hence, if $\mathcal{M}$ is a refinement ideal, $\left\{\Sigma_{E} \mid E \in \mathcal{M}\right\}$ is a directed family of subalgebras of $\mathcal{P}(S)$ under inclusion, whence, its union is also a subalgebra.

Now let $A$ be a refinement ideal in the sense of probabilistic models, i.e., $\mathcal{M}(A)$ is a refinement ideal with respect to $\Omega(A)$. For every $\alpha \in \Omega(A)$, define

$$
\widehat{\alpha}(\widehat{a})=\alpha(a)
$$

for every $\widehat{a} \in \Sigma$. 
Lemma A.6 $\widehat{\alpha}$ is well-defined, and a finitely-additive probability measure on $\Sigma$.

Proof: Let $a \subseteq E \in \mathcal{M}$ and $b \subseteq F \in \mathcal{M}$. We want to show that if $\widehat{a}=\widehat{b}$, then $\alpha(a)=\alpha(b)$. Let $G$ refine both $E$ and $F$. There are canonical surjections $e: G \rightarrow E$ and $f: G \rightarrow F$, namely $e(z)=x$ where $x$ is the unique cell of $E$ containing $z \in G$, and similarly for $f$. Let $a_{1}=e^{-1}(a)$ and $b_{1}=f^{-1}(b)$. Then $\widehat{a_{1}}=\widehat{a}=\widehat{b}=\widehat{b_{1}}$. Since $\widehat{ }{ }^{-}$is injective on $\mathcal{P}(G), a_{1}=b_{1}$. Since $A$ is a refinement ideal with respect to $\Omega(A)$, $\alpha(a)=\alpha\left(a_{1}\right)=\alpha\left(b_{1}\right)=\alpha(b)$.

This shows that $\widehat{\alpha}$ is well defined. To see that it's additive, let $a \subseteq E \in \mathcal{M}(A), b \subseteq F \in \mathcal{M}(A)$, with $\widehat{a} \cap \widehat{b}=\emptyset$. Choosing $G$ a common refinement of $E$ and $F$, we have $a_{1}, b_{1} \subseteq G$ with $\widehat{a}_{1}=\widehat{a}$ and $\widehat{b}_{1}=\widehat{b}$, so $\widehat{a}_{1} \cap \widehat{b}_{1}=\emptyset$, whence, $a_{1} \cap b_{1}=\emptyset$. It follows that

$$
\begin{aligned}
\widehat{\alpha}(\widehat{a} \cup \widehat{b})=\widehat{\alpha}\left(\widehat{a_{1} \cup a_{2}}\right)=\alpha\left(a_{1} \cup a_{2}\right) & =\alpha\left(a_{1}\right)+\alpha\left(a_{2}\right) \\
& =\widehat{\alpha}\left(\widehat{a}_{1}\right)+\widehat{\alpha}\left(\widehat{b}_{1}\right)=\widehat{\alpha}(\widehat{a})+\widehat{\alpha}(\widehat{b}) .
\end{aligned}
$$

Proposition A.7 If $A$ is a locally finite refinement ideal and $\Omega(A)$ separates compatible events, then there is a measurable space $(S, \Sigma)$ and an embedding $\mathcal{M}(A) \rightarrow \mathcal{D}(S, \Sigma)$ such that every probability weight in $\Omega$ extends to a finitely-additive probability measure on $(S, \Sigma)$. Conversely, if $A$ is unital and locally finite and admits such an embedding, then $A$ embeds in a refinement ideal without loss of states.

Proof: The forward implication follows from Lemmas A.4, A.5 and A.6, while the reverse implication is more or less obvious.

To this extent, then, the existence of common refinements is the key classical postulate.

\section{B Semiclassical test spaces}

Recall that $\mathcal{M}$ is semiclassical iff distinct tests in $\mathcal{M}$ are disjoint. Evidently, such a test space has a wealth of dispersion-free states, as we can simply choose an element $x_{E}$ from each test $E \in \mathcal{M}$ and set $\delta(x)=1$ if $x=x_{E}$ for the unique test containing $x$, and 0 otherwise. In fact, the dispersion-free states are exactly the pure states:

Lemma B.1 Let $K_{i}$ be an indexed family of convex sets, and let $\alpha=\left(\alpha_{i}\right) \in K=\prod_{i \in I} K_{i}$. Then $\alpha$ is pure iff each $\alpha_{i}$ is pure.

Proof: Suppose that for some $j \in I, \alpha_{j}$ is not pure. Then there exist distinct points $\beta_{j}, \gamma_{j} \in K_{j}$ with $\alpha_{j}=t \beta_{j}+(1-t) \gamma_{j}$ for some $t \in(0,1)$. Define $\widehat{\beta}, \widehat{\gamma} \in K$ by setting

$$
\widehat{\beta}_{i}=\left\{\begin{array}{cc}
\alpha_{i} & i \neq j \\
\beta_{j} & i=j
\end{array} \text { and } \widehat{\gamma}_{i}= \begin{cases}\alpha_{i} & i \neq j \\
\gamma_{j} & i=j\end{cases}\right.
$$

Then we have $t \widehat{\beta}+(1-t) \widehat{\gamma}=\alpha$, so $\alpha$ is not pure either. The converse is clear.

Corollary B.2 If $\mathcal{M}$ is semiclassical, then $\operatorname{Pr}(\mathcal{M})_{\text {ext }}=\operatorname{Pr}(\mathcal{M}) d f$.

Proof: Since $\mathcal{M}$ is semiclassical, $\operatorname{Pr}(\mathcal{M}) \simeq \Pi_{E \in \mathcal{M}} \Delta(E)$. 


\section{Constructing fully $G$-symmetric models}

This appendix collects more technical material on the construction of fully symmetric models. In particular, we prove Theorem 4.7, which for convenience we restate below as Proposition C.2.

As discussed in Section 4.2, we are given a set $E$ (which we wish to regard as the outcome-set of an experiment), and a group $H$ acting transitively on $E$. We are also given a set $\Omega$ of physical states (those of the system to which the experiment pertains), and a function

$$
p: \Omega \times E \rightarrow[0,1]
$$

assigning a probability weight $\widehat{\alpha}=p(\alpha, \cdot)$ on $E$ to each state $\alpha \in \Omega$. We are also given a group $G$ of "physical symmetries" acting on $\Omega$ on the right, plus a subgroup $H$ of $G$ acting on $E$ (on the left), in such a way that

$$
p(\alpha h, x)=p(\alpha, h x)
$$

for all $h \in H$. For each $\alpha \in \Omega$, we write $\widehat{\alpha}$ both for the mapping $G \mapsto \Delta(E)$ given by $\widehat{\alpha}(g)(x)=p(\alpha g, x)$, and for the mapping $G \times E \rightarrow[0,1]$ given by $\widehat{\alpha}(g, x)=\widehat{\alpha}(g)(x)$, whichever is more convenient. Thus, $\widehat{\alpha g_{1}}\left(g_{2}\right)=\widehat{\alpha}\left(g_{1} g_{2}\right)$ for all $g_{1}, g_{2} \in G$, and $\widehat{\alpha}(g h, x)=\widehat{\alpha}(g, h x)$ for all $g \in G, h \in H$ and $x \in E$. We assume that the mapping $\alpha \mapsto \widehat{\alpha}$ is injective, so that $\alpha$ is determined by the function $\widehat{\alpha}$.

Given this data, choose and fix an outcome $x_{o} \in E$, and let

$$
\mathbb{K}:=\left\{k \in G \mid \forall \alpha \in \Omega p\left(\alpha k, x_{o}\right)=p\left(\alpha, x_{o}\right)\right\} .
$$

Equivalently, $k \in \mathbb{K}$ iff $\widehat{\alpha}\left(g k, x_{o}\right)=\widehat{\alpha}\left(g, x_{o}\right)$ for all $g \in G$. Notice that the stabilizer, $H_{o}$, of $x_{o}$ in $H$ is a subset of $\mathbb{K}$; in particular, $\mathbb{K}$ is nonempty.

Lemma C.1 $\mathbb{K} \leq G$, and $\mathbb{K} \cap H=H_{o}$.

Proof: Let $k, k^{\prime} \in \mathbb{K}$. Then for all $g \in G$ and $\alpha \in \Omega$, we have $\widehat{\alpha}\left(g k k^{\prime}, x_{o}\right)=\widehat{\alpha}\left(g k, x_{o}\right)=\widehat{\alpha}\left(g, x_{o}\right)$ so $k k^{\prime} \in \mathbb{K}$; also

$$
\widehat{\alpha}\left(g k^{-1}, x_{o}\right)=\widehat{\alpha}\left(\left(g k^{-1}\right) k, x_{o}\right)=\widehat{\alpha}\left(g, x_{o}\right)
$$

so $k^{-1} \in \mathbb{K}$. Thus, $\mathbb{K}$ is a subgroup of $G$. For the second statement, $h \in \mathbb{K} \cap H$ implies that, for all $\alpha$ and all $g$,

$$
\widehat{\alpha}\left(g, x_{o}\right)=\widehat{\alpha}\left(g h, x_{o}\right)=\widehat{\alpha}\left(g, h x_{o}\right) .
$$

Since the functions $\widehat{\alpha}(g)$ separate points of $E, h x_{o}=x_{o}$, so $h \in H_{o}$ On the other hand, if $h \in H_{o}$, then $\widehat{\alpha}\left(g h, x_{o}\right)=\widehat{\alpha}\left(g, h x_{o}\right)=\widehat{\alpha}\left(g, x_{o}\right)$, so $h \in \mathbb{K}$.

We now prove Theorem 4.7, showing that any choice of a subgroup $K$ between $H_{o}$ and $\mathbb{K}$ generates a fully $G$-symmetric test space, having $\Omega$ as its state space, in a canonical manner. For convenience, we restate this result here:

Proposition C.2 With notation as above, suppose $H$ acts transitively on E. Let $K$ be any subgroup of $G$ with $H_{o} \leq K \leq \mathbb{K}$. Set $X:=G / K$, and let $x_{g}:=g K \in X$ for all $g \in G$. Then there is a well-defined $H$-equivariant injection $\phi: E \rightarrow X$ given by $\phi\left(h x_{o}\right)=x_{h}$ for all $h \in H$. Moreover, identifying $E$ with $\phi(E) \subseteq X$,

(a) $\mathcal{M}:=\{g \phi(E) \mid g \in G\}$ is a G-symmetric test space;

(b) For every $\alpha \in \Omega, \underset{\sim}{\alpha}\left(x_{g}\right):=\widehat{\alpha}\left(g, x_{o}\right)$ is a well-defined probability weight on $\mathcal{M}$;

(c) The mapping $\alpha \mapsto \alpha$ is a G-equivariant affine injection, where $G$ acts on $\Delta(E)^{G}$ on the right. 
Proof: For convenience, let us write $x_{g}$ for the left coset $g K$ in $X=G / K$. Then the mapping $\phi: E \rightarrow X$ above is given by

$$
\phi: h x_{o} \mapsto x_{h}
$$

To see this is well-defined, let $h, h^{\prime} \in H$ with $h x_{o}=h^{\prime} x_{o}$ : then $h^{-1} h^{\prime} \in H_{o} \leq K$, so that $x_{h}=x_{h^{\prime}}$. Conversely, if $x_{h}=x_{h^{\prime}}$, we have $h^{-1} h^{\prime} \in S_{o}$ whence $h x_{o}=h^{\prime} x_{o}$, and $\phi$ is injective. If $x=h x_{o}$, then $\phi\left(h^{\prime} x\right)=h^{\prime} h K=h^{\prime} x_{h}=h \phi(x)$, so $\phi$ is $H$-equivariant. This disposes of (a). Now let $\mathcal{M}=\{g \widetilde{E} \mid g \in G\}$. Since $X$ is a $G$-set, this gives us a $G$ test space - a symmetric one, as $G$ acts transitively on $\mathcal{M}$ and $H$ acts transitively on $\phi(E) \in \mathcal{M}$. For $\alpha \in \Omega$, set $\underset{\sim}{\alpha}\left(x_{g}\right)=\widehat{\alpha}\left(g, x_{o}\right)$. This is well-defined, since if $x_{g}=x_{g^{\prime}}$, then $g^{\prime}=g k$ for some $k \in K$, and thus

$$
\underset{\sim}{\alpha}\left(x_{g^{\prime}}\right)=\underset{\sim}{\alpha}\left(x_{g k}\right)=\widehat{\alpha}\left(g k, x_{o}\right)=\widehat{\alpha}\left(g, x_{o}\right)=\underset{\alpha}{\alpha}\left(x_{g}\right) .
$$

To see that $\underset{\sim}{\alpha}$ is a probability weight on $\mathcal{M}$, for each $x \in E$, choose $h_{x} \in H$ with $h_{x} x_{o}=x$ (recalling here that $H$ acts transitively on $E$ ). Then for all $g \in G$ we have

$$
\underset{\sim}{\alpha}(g \phi(x))=\underset{\sim}{\alpha}\left(x_{g h_{x}}\right)=\widehat{\alpha}\left(g h_{x}, x_{o}\right)=\widehat{\alpha}\left(g, h_{x} x_{o}\right)=\widehat{\alpha}(g, x) .
$$

Thus,

$$
\sum_{x \in E} \underset{\sim}{\alpha}(g \phi(x))=\sum_{x \in E} \widehat{\alpha}(g, x)=1 .
$$

The mapping $\alpha \mapsto \underset{\sim}{\alpha}$ is obviously affine, and is injective by our assumption that $\alpha \mapsto \widehat{\alpha}$ is injective. To see that it is equivariant, note that for all $g, l \in G$ and $\alpha \in \Omega$,

$$
\left.(\underline{\alpha g})\left(x_{l}\right)=\widehat{(\alpha g}\right)\left(l, x_{o}\right)=\widehat{\alpha}\left(g l, x_{o}\right)=\underline{\alpha}\left(g l x_{o}\right)=\underline{\alpha}\left(g x_{l}\right)=(\underline{\alpha} \circ g)\left(x_{l}\right) .
$$

Thus, $(\mathcal{M}, \Omega)$, where $\underset{\sim}{\Omega}=\{\alpha \underset{\sim}{\alpha} \mid \alpha \in \Omega\}$, is a $G$-symmetric model with state space isomorphic to $\Omega$.

Remarks:

(1) If $H_{o} \leq K \leq K^{\prime} \leq \mathbb{K}$, we obtain a $G$-equivariant, surjective outcome-preserving interpretation

$$
\phi_{K^{\prime}, K}: \mathcal{M}_{K} \rightarrow \mathcal{M}_{K^{\prime}}
$$

given by $\phi_{K^{\prime}, K}(g K)=g K^{\prime}$ for all $g \in G$. So taking $K=H_{o}$ gives in this sense the least constrained symmetric $G$-test space containing $E$ (in such a way as to extend the action of $H$ on $E$ ), while $K=\mathbb{K}$ gives the most constrained. Note all such test spaces have tests of the same size, namely $|E|$, provided that the set of probability weights $\widehat{\alpha}(g)$, with $\alpha$ ranging over $\Omega$ and $g$ ranging over $G$, is large enough to separate points of $E$.

(2) Accordingly, all probability weights on $\mathcal{M}_{K^{\prime}}$ pull back to probability weights on $\mathcal{M}_{K}$ via $\phi_{K, K^{\prime}}^{*}$, which is an injective $G$-equivariant affine mapping. Writing $\Omega_{K}:=\operatorname{Pr}\left(\mathcal{M}_{K}\right)$ for the convex set of all probability weights on $\mathcal{M}_{K}$, we have $\Omega \subseteq \Omega_{K}$ (where we identify $\Omega$ with its image $\Omega$ ). Thus, in particular, we can replace $\Omega$ with $\operatorname{Pr}\left(\mathcal{M}_{K}\right)$ to obtain a larger state space with respect to which the same construction works.

Given the $H$-set $E$, we can obtain the initial data for this construction as follows. Let $G$ be any group containing $H$ as a subgroup, and let

$$
G \times{ }_{H} E=(G \times E) / \sim
$$

where $\sim$ is the equivalence relation defined by $(g, x) \sim(h, y)$ iff there exists some $s \in H$ with $h=g s$ and $s y=x$. Letting $[g, x]$ denote the equivalence class of $(g, x)$, we have

$$
[g, x]=\left[g s, s^{-1} x\right] \text { i.e., }[g s, x]=[g, s x]
$$

for all $g \in G, x \in E$ and $s \in H$. Note that $G \times_{H} E$ is a $G$-set, with action defined by

$$
g[h, x]=[g h, x]
$$


which is well-defined because $\left[g h s, s^{-1} x\right]=[g h, x]$ for all $s \in H$. Now let

$$
[g, E]:=\{[g, x] \mid x \in E\}:
$$

then if $[h, x] \in[g, E]$, we have $[h, x]=[g, y]$ for some $y \in E$, whence, there is some $s \in S$ with $h=g s$ and $s y=x$. Then for all $z \in E$, say $z=s^{\prime} x$, we have $[h, z]=\left[g s, s^{\prime} x\right]=\left[g, s s^{\prime} x\right] \in[g, E]$. That is, $[h, E] \subseteq[g, E]$. By the same token, $[g, E] \subseteq[h, E]$. In other words, the sets $[g, E]$ paritition $G \times_{H} E$. With this observation, it is easy to prove the following

Lemma C.3 With notation as in Proposition C.2, $X_{H_{o}} \simeq G \times_{H_{o}} E$, and $\mathcal{M}_{H_{o}} \simeq\{[g, E] \mid g \in E\}, a$ semiclassical test space, independent of $\Omega$.

Proof: Let $\phi: G \times E \rightarrow G / H_{o}=X_{H_{o}}$ be given by

$$
\phi\left(g, s x_{o}\right)=g s H_{o} .
$$

To see that this is well-defined, note that if $s_{1} x_{o}=s_{2} x_{o}$, then $s_{2}^{-1} s_{1}=: s \in H_{o}$, and $g s_{1} H_{o}=g s_{2} s H_{o}=$ $g s_{2} H_{o}$. Next, observe that $\phi\left(g_{1}, s_{1} x_{o}\right)=\phi\left(g_{2}, s_{2} x_{o}\right)$ iff $g_{1} s_{1} H_{o}=g_{2} s_{2} H_{o}$ iff $g_{2} s_{2}=g_{1} s_{1} s$ for some $s \in H_{o}$, whence, $\left[g_{2}, s_{2} x_{o}\right]=\left[g_{2} s_{2}, x_{o}\right]=\left[g_{1} s_{1} s, x_{o}\right]=\left[g_{1}, s_{1} x_{o}\right]$. Thus, passing to the quotient set $G \times_{H_{o}} E$ gives us an equivariant bijection. Since $\{[g, E] \mid g \in G\}$ is a partition of $G \times{ }_{H_{o}} E$, we have a semiclassical tets space, as advertised.

Notice that the stabilizer of $[e, E]$ in $G$ is exactly $H_{o}$. Now choosing any $G$-invariant set of probability weights on $\mathcal{M}_{H_{o}}$ - say, the orbit of any given probability weight — we have a natural $G$-equivariant injection $\Omega \rightarrow \Delta(E)^{G}$ given by

$$
\alpha \mapsto \widehat{\alpha}: \widehat{\alpha}(g)(x)=\alpha[g, x]
$$

for all $\alpha \in \Omega$. The construction now proceeds as above for any choice of subgroup $K$ of $G$ with $H_{o} \leq K \leq \mathbb{K}$, where $\mathbb{K}$ is determined by $\Omega$ as in Equation (6). We can regard $K$ as parametrizing the possible fully $G$ symmetric models containing $E$ as a test, and having dynamical group $G$. As shown above, if $K=H_{o}$, the stabilizer of $x_{o}$ in $H$, then $\mathcal{M}$ is semiclassical. Larger choices of $K$ further constrain the structure of $\mathcal{M}$, enforcing outcome-identifications between tests that, in turn, constrain any further enlargement of the state space that we might contemplate. 